\title{
A BOUND FOR CASTELNUOVO-MUMFORD REGULARITY BY DOUBLE POINT DIVISORS
}

\author{
SIJONG KWAK AND JINHYUNG PARK
}

\begin{abstract}
Let $X \subseteq \mathbb{P}^{r}$ be a non-degenerate smooth projective variety of dimension $n$, codimension $e$, and degree $d$ defined over an algebraically closed field of characteristic zero. In this paper, we first show that $\operatorname{reg}\left(\mathcal{O}_{X}\right) \leq d-e$, and classify the extremal and the next to extremal cases. Our result reduces the Eisenbud-Goto regularity conjecture for the smooth case to the problem finding a Castelnuovo-type bound for normality. It is worth noting that McCullough-Peeva recently constructed counterexamples to the regularity conjecture by showing that $\operatorname{reg}\left(\mathcal{O}_{X}\right)$ is not even bounded above by any polynomial function of $d$ when $X$ is not smooth. For a normality bound in the smooth case, we establish that $\operatorname{reg}(X) \leq n(d-2)+1$, which improves previous results obtained by Mumford, Bertram-Ein-Lazarsfeld, and Noma. Finally, by generalizing Mumford's method on double point divisors, we prove that $\operatorname{reg}(X) \leq$ $d-1+m$, where $m$ is an invariant arising from double point divisors associated to outer general projections. Using double point divisors associated to inner projection, we also obtain a slightly better bound for $\operatorname{reg}(X)$ under suitable assumptions.
\end{abstract}

\section{INTRODUCTION}

Throughout the paper, we work over an algebraically closed field of characteristic zero. Let $H$ be a very ample divisor on a smooth projective variety $X$. A celebrated vanishing theorem of Serre asserts that there exists an integer $k_{0}=k_{0}(H)$ such that if $k \geq k_{0}$, then

$$
H^{i}\left(X, \mathcal{O}_{X}((k-i) H)\right)=0 \text { for } i>0 .
$$

It is a natural problem to find an upper bound for $k_{0}(H)$ in terms of geometric invariants of $X$ and $H$. The first main result of this paper provides a sharp effective upper bound for $k_{0}(H)$ using the delta genus

$$
\Delta(X, H):=H^{\operatorname{dim}(X)}+\operatorname{dim}(X)-h^{0}\left(X, \mathcal{O}_{X}(H)\right)
$$

of the polarized pair $(X, H)$.

Theorem A. Let $X$ be a smooth projective variety, and $H$ be a very ample divisor on $X$. If $k \geq \Delta(X, H)+1$, then

$$
H^{i}\left(X, \mathcal{O}_{X}((k-i) H)\right)=0 \text { for } i>0 \text {. }
$$

In particular, we have

$$
\chi(X, k H)=h^{0}(X, k H) \text { for } k \geq \Delta(X, H) .
$$

Date: September 7, 2018.

2010 Mathematics Subject Classification. Primary 14N05, 13D02, 14N25; Secondary 51N35.

Key words and phrases. Castelnuovo-Mumford regularity, regularity conjecture, double point divisor, projection, vanishing theorem.

S. Kwak was supported by Basic Science Research Program through the National Research Foundation of Korea (NRF) funded by the Ministry of Science and ICT (2015R1A2A2A01004545). 
If $X \subseteq \mathbb{P}^{r}$ is a rational normal scroll of dimension $n$ and $H$ is its hyperplane section, then $\Delta(X, H)=0$ and $H^{n}\left(X, \mathcal{O}_{X}(-n H)\right) \neq 0$. This shows that our bound in Theorem A is sharp. Furthermore, we classify polarized pairs $(X, H)$ such that $H^{i}\left(X, \mathcal{O}_{X}((\Delta(X, H)-i) H)\right) \neq 0$ for some $i>0$ in Theorem B.

We now turn to the regularity conjecture, which is closely related to the effective Serre vanishing problem. The Castelnuovo-Mumford regularity of an embedded projective variety $X \subseteq \mathbb{P}^{r}$ is defined as

$$
\operatorname{reg}(X):=\min \left\{k+1 \mid X \subseteq \mathbb{P}^{r} \text { is } k \text {-normal and } \mathcal{O}_{X} \text { is } k \text {-regular }\right\} .
$$

We denote by $\operatorname{reg}\left(\mathcal{O}_{X}\right)$ the minimum $k$ such that $\mathcal{O}_{X}$ is $k$-regular, i.e., $H^{i}\left(X, \mathcal{O}_{X}(k-i)\right)=0$ for $i>0$. It is a fundamental problem, initiated by Castelnuovo, Mumford, Gruson-LazarsfeldPeskine, etc., to find an explicit upper bound for $\operatorname{reg}(X)$ in terms of geometric invariants of $X \subseteq \mathbb{P}^{r}$. One particular reason to consider this problem is that $\operatorname{reg}(X)$ gives an upper bound for the degree of defining equations of $X$ in $\mathbb{P}^{r}$ because reg $(X)$ is the maximal degree of syzygies among minimal generators of the defining ideal $I_{X \mid \mathbb{P}^{r}}$. We also remark that $\operatorname{reg}\left(\mathcal{O}_{X}\right)$ is the maximal degree of syzygies of the section $\operatorname{ring} R\left(X, \mathcal{O}_{X}(1)\right):=\bigoplus_{k \geq 0} H^{0}\left(X, \mathcal{O}_{X}(k)\right)$. An optimal Castelnuovo-type bound for the regularity was suggested by Eisenbud-Goto [EG] (see also [GLP, Section 4]) as follows:

Regularity Conjecture. Let $X \subseteq \mathbb{P}^{r}$ be a non-degenerate projective variety of degree $d$ and codimension e. Then we have

$$
\operatorname{reg}(X) \leq d-e+1
$$

This conjecture has been a long-standing and challenging problem, and there has been a considerable amount of interesting partial results. Castelnuovo [C] carried out the fundamental work in this direction for smooth space curves, and Gruson-Lazarsfeld-Peskine [GLP] completely settled the regularity conjecture for integral curves. Pinkham [P] and Lazarsfeld [L1] verified the conjectured bound for the regularity for smooth surfaces, and Niu [Ni] proved the same result for mildly singular surfaces. For smooth threefolds, Kwak [K1], [K2] obtained a weaker bound $\operatorname{reg}(X) \leq d-e+2$ for general case and a sharp bound $\operatorname{reg}(X) \leq d-1$ for codimension 2 case. This result is extended to threefolds with rational singularities by Niu-Park [NP2]. Slightly weaker bounds for lower dimensional smooth varieties were established by Kwak [K3]. It is also an important problem to classify varieties with the maximal or the next to maximal regularity. The classification of projective curves with maximal regularity is given in [GLP], but almost nothing is known in higher dimensions.

To solve the regularity conjecture, it is sufficient to show that

(1) $X \subseteq \mathbb{P}^{r}$ is $(d-e)$-normal, and

(2) $\operatorname{reg}\left(\mathcal{O}_{X}\right) \leq d-e$.

Until recently, most results on the regularity conjecture have been centered around the problem (1). Notice that if the regularity conjecture holds in dimension $n$, then the inequality (2) in dimension $n+1$ follows (see Lemma 2.1). Thus the inequality (2) holds for any projective surfaces and for projective threefolds with isolated singularities (see [NP1, Proposition 2.3]). For the curve case, we have a stronger result: $\operatorname{reg}\left(\mathcal{O}_{C}\right) \leq\left\lfloor\frac{d-1}{e}\right\rfloor+1$ (see Corollary 2.3).

It turns out that the problem (2) is evidently nontrivial and important. Recently, McCulloughPeeva $[\mathrm{MP}]$ constructed counterexamples to the regularity conjecture. Their counterexamples actually show that $\operatorname{reg}\left(\mathcal{O}_{X}\right)$ is not even bounded above by any polynomial functions of the 
degree $d$ when $X \subseteq \mathbb{P}^{r}$ is a highly singular projective variety. It is worth to mention that the regularity conjecture is still open for normal projective varieties.

In this paper, we verify the inequality $(2) \operatorname{reg}\left(\mathcal{O}_{X}\right) \leq d-e$ when $X$ is a smooth projective variety of arbitrary dimension. We also classify the extremal and the next to extremal cases.

Theorem B. Let $X \subseteq \mathbb{P}^{r}$ be a non-degenerate smooth projective variety of degree $d$ and codimension e. Then we have the following:

(a) $\operatorname{reg}\left(\mathcal{O}_{X}\right) \leq d-e$.

(b) $\operatorname{reg}\left(\mathcal{O}_{X}\right)=d-e$ if and only if $X \subseteq \mathbb{P}^{r}$ is a hypersurface or a linearly normal variety with $d=e+1$ or $e+2$.

(c) $\operatorname{reg}\left(\mathcal{O}_{X}\right)=d-e-1$ if and only if $X \subseteq \mathbb{P}^{r}$ is an isomorphic projection of a projective variety in $(a)$ at one point, a linearly normal variety with $d=e+3$ and $e \geq 2$, or $a$ complete intersection of type $(2,3)$.

Observe that $\Delta\left(X, \mathcal{O}_{X}(1)\right)+1 \leq d-e$ and the equality holds when $X \subseteq \mathbb{P}^{r}$ is linearly normal. By Mumford's regularity theorem, if $\mathcal{O}_{X}$ is $k$-regular, then it is $(k+1)$-regular. Thus Theorem B $(a)$ is equivalent to Theorem A, and can be rephrased as

$$
\operatorname{reg}\left(\mathcal{O}_{X}\right) \leq \Delta\left(X, \mathcal{O}_{X}(1)\right)+1
$$

We emphasize that the delta genus $\Delta\left(X, \mathcal{O}_{X}(1)\right)$ is not an extrinsic invariant depending on the given embedding $X \subseteq \mathbb{P}^{r}$ but an intrinsic invariant depending only on the variety $X$ and the very ample line bundle $\mathcal{O}_{X}(1)$.

The complete classification of projective varieties appeared in Theorem B $(b)$ and $(c)$ is given in Remark 3.8. By the classification, it is easy to check that those varieties satisfy $\operatorname{reg}(X)=d-e+1$ or $\operatorname{reg}(X)=d-e$.

A classical approach for the study of the projective geometry of an embedded projective variety $X \subseteq \mathbb{P}^{r}$ is to consider a general outer projection $\pi: X \rightarrow \bar{X} \subseteq \mathbb{P}^{n+1}$ onto a hypersurface of degree $d$. Then $\pi$ is a birational morphism, and the non-isomorphic locus of $\pi$ gives rise to an effective divisor linearly equivalent to the double point divisor from outer projection

$$
D_{\text {out }}:=-K_{X}+(d-n-2) H,
$$

where $H$ is a hyperplane section of $X \subseteq \mathbb{P}^{r}$. By varying projection centers, Mumford [BM] proved that $D_{\text {out }}$ is base point free. In [No2], Noma extended Mumford's result to the inner projection case. Let $\pi: X \rightarrow \bar{X} \subseteq \mathbb{P}^{n+1}$ be a general inner projection onto a hypersurface of degree $d-e+1$. Similarly as in the outer projection case, we define the double point divisor from inner projection

$$
D_{i n n}:=-K_{X}+(d-n-e-1) H .
$$

The main result of [No2] says that $D_{i n n}$ is semiample unless $X \subseteq \mathbb{P}^{r}$ is a scroll over a curve, the second Veronese surface, or a Roth variety. When $D_{i n n}$ is semiample, it follows from Kodaira vanishing theorem that $\operatorname{reg}\left(\mathcal{O}_{X}\right) \leq d-e$. This bound can also be easily checked for the second Veronese surface and Roth varieties. In Section 3, we show a sharp bound for $\operatorname{reg}\left(\mathcal{O}_{X}\right)$ when $X \subseteq \mathbb{P}^{r}$ is a scroll over a curve using Castelnuovo's genus bound and Ionescu-Toma's result [IT]. Our proof involves complicated calculations, but it is a natural generalization of the arguments in the curve case.

By Theorem B, the regularity conjecture for the smooth case is reduced to finding a sharp Castelnuovo-type bound for normality. Note that the first general result on a bound for $\operatorname{reg}(X)$ 
was obtained by Mumford $[\mathrm{BM}]$ : if $X \subseteq \mathbb{P}^{r}$ is a non-degenerate smooth projective variety of dimension $n$, codimension $e$, and degree $d$, then

$$
\operatorname{reg}(X) \leq(n+1)(d-2)+2 .
$$

Bertram-Ein-Lazarsfeld [BEL] and Noma [No3] refined Mumford's result as

$$
\operatorname{reg}(X) \leq e(d-e)+1 \text { if } e \leq n \text { and } \operatorname{reg}(X) \leq(n+1)(d-n-1)+1 \text { if } e \geq n+1 .
$$

We remark that not every smooth projective variety of dimension $n$ can be embedded in $\mathbb{P}^{2 n}$. Moreover, by Barth-Larsen theorem, if $e \leq n-1$, then $X$ is simply connected, and if $e \leq n-2$, then $\operatorname{Pic}(X)$ is generated by the hyperplane section. Thus we may assume in general that $e \geq n+1$, and then, the previous best bound is essentially that

$$
\operatorname{reg}(X) \leq(n+1) d+\alpha_{n},
$$

where $\alpha_{n}$ is a constant depending only on $n$. Our next aim is to improve the previous results.

Theorem C. Let $X \subseteq \mathbb{P}^{r}$ be a non-degenerate smooth projective variety of dimension $n$, codimension $e \geq 2$, and degree $d$. Then we have

$$
\operatorname{reg}(X) \leq n(d-2)+1=n d-2 n+1
$$

The main ingredients of the proof are the classical methods of Castelnuovo [C] and Mumford $[\mathrm{BM}],[\mathrm{M}]$ and a vanishing theorem of de Fernex-Ein [dFE].

Finally, to get a better normality bound, we extend Mumford's method on double point divisors in $[\mathrm{BM}]$ as Lemma 5.2, and then, show a Castelnuovo-Mumford regularity bound for smooth varieties using an invariant arising from double point divisors. Let $V_{\text {out }}$ be a subspace of $H^{0}\left(X, \mathcal{O}_{X}\left(D_{\text {out }}\right)\right)$ spanned by geometric sections, which are global sections of $\mathcal{O}_{X}\left(D_{\text {out }}\right)$ whose zero loci are non-isomorphic loci of general outer projections, and $c_{k}$ be the codimension of the image of the multiplication map $V_{\text {out }} \otimes H^{0}\left(X, \mathcal{O}_{X}(k)\right) \rightarrow H^{0}\left(X, \mathcal{O}_{X}\left(D_{\text {out }}+k H\right)\right)$ for any integer $k \geq 0$. We define

$$
m:=\min \left\{c_{k}+k \mid-2 K_{X}+(d-2 n-3+k) H \text { is nef }\right\} .
$$

Theorem D. Let $X \subseteq \mathbb{P}^{r}$ be a smooth projective variety of degree $d$. Then we have

$$
\operatorname{reg}(X) \leq d-1+m
$$

Concerning a bound for $m$, we show that $-2 K_{X}+(d-2 n-3+k) H$ is nef for $k \geq d-1$ and $c_{k}=0$ for $k \geq n(d-3)$ in Propositions 5.4 and 5.5. See Remark 5.6 for further discussions.

We also have a similar result for the inner projection case. Let $V_{i n n}$ be a subspace of $H^{0}\left(X, \mathcal{O}_{X}\left(D_{i n n}\right)\right)$ spanned by geometric sections, and $c_{k}^{\prime}$ be the codimension of the image of the map $V_{\text {inn }} \otimes H^{0}\left(X, \mathcal{O}_{X}(k)\right) \rightarrow H^{0}\left(X, \mathcal{O}_{X}\left(D_{i n n}+k H\right)\right)$ for any integer $k \geq 0$. We define

$$
m^{\prime}:=\min \left\{c_{k}^{\prime}+k \mid-2 K_{X}+(d-2 n-e-2+k) H \text { is nef }\right\} .
$$

Theorem E. Let $X \subseteq \mathbb{P}^{r}$ be a non-degenerate smooth projective variety of dimension $n \geq 2$, codimension $e \geq 2$ and degree $d \geq e+3$ such that it is neither a scroll over a smooth projective curve, the second Veronese surface, a Roth variety, nor a complete intersection of type $(2,3)$. Suppose that $V_{\text {inn }}$ is base point free and the multiplication map

$$
H^{0}\left(\mathbb{P}^{r}, \mathcal{O}_{\mathbb{P}^{r}}(i)\right) \otimes H^{0}\left(X, \mathcal{O}_{X}\left(K_{X}+(n-1) H\right)\right) \longrightarrow H^{0}\left(X, \mathcal{O}_{X}\left(K_{X}+(n-1+i) H\right)\right)
$$

is surjective for $i=1,2$. Then we have

$$
\operatorname{reg}(X) \leq d-e-1+m^{\prime} .
$$


We remark that if the conditions of Theorem E are fulfilled and $m^{\prime} \leq 2$, then the conjectured bound $\operatorname{reg}(X) \leq d-e+1$ holds.

The rest of the paper is organized as follows: We start in Section 2 by recalling relevant basic facts including the positivity properties of double point divisors. Section 3 is devoted to the proofs of Theorems A and B. In Section 4, we prove Theorem C. Finally, in Section 5, we generalize Mumford's method, and show Theorems D and E.

\section{Preliminaries}

In this section, we collect basic definitions and facts that are useful throughout the paper.

2.1. Castelnuovo-Mumford regularity. Let $X$ be a projective variety, and $L$ be a very ample line bundle on $X$. A coherent sheaf $\mathcal{F}$ on $X$ is said to be $k$-regular with respect to $L$ in the sense of Castelnuovo-Mumford if $H^{i}\left(X, \mathcal{F} \otimes L^{\otimes k-i}\right)=0$ for all $i>0$. By Mumford's regularity theorem ([L2, Theorem 1.8.5]), if $\mathcal{F}$ is $k$-regular, then $\mathcal{F}$ is $(k+1)$-regular. We say that an embedded projective variety $X \subseteq \mathbb{P}^{r}$ is $k$-regular if the ideal sheaf $\mathcal{I}_{X \mid \mathbb{P}^{r}}$ is $k$-regular with respect to $\mathcal{O}_{\mathbb{P}^{r}}(1)$. Note that $X \subseteq \mathbb{P}^{r}$ is $k$-regular if and only if

(1) $X \subseteq \mathbb{P}^{r}$ is $(k-1)$-normal, i.e., the natural restriction map

$$
H^{0}\left(\mathbb{P}^{r}, \mathcal{O}_{\mathbb{P}^{r}}(k-1)\right) \rightarrow H^{0}\left(X, \mathcal{O}_{X}(k-1)\right)
$$

is surjective, and

(2) $\mathcal{O}_{X}$ is $(k-1)$-regular with respect to $\mathcal{O}_{X}(1)$.

The Castelnuovo-Mumford regularity of $X$, denoted by $\operatorname{reg}(X)$, is the least integer $k$ such that $X \subseteq \mathbb{P}^{r}$ is $k$-regular. We also denote by $\operatorname{reg}\left(\mathcal{O}_{X}\right)$ the least integer $k$ such that $\mathcal{O}_{X}$ is $k$-regular. We refer to [L2, Section 1.8] for more details.

Lemma 2.1 (cf. [NP1, Lemma 2.2]). Let $X \subseteq \mathbb{P}^{r}$ be a non-degenerate projective variety of dimension $n \geq 2$, and $Y \subseteq \mathbb{P}^{r-1}$ be a general hyperplane section. Fix integers $k_{0}$ and $i \geq 1$. Suppose that

$$
\left\{\begin{array}{cl}
Y \subseteq \mathbb{P}^{r-1} \text { is } k \text {-normal and } H^{1}\left(Y, \mathcal{O}_{Y}(k)\right)=0 & \text { if } i=1 \\
H^{i-1}\left(Y, \mathcal{O}_{Y}(k)\right)=H^{i}\left(Y, \mathcal{O}_{Y}(k)\right)=0 & \text { if } i \geq 2
\end{array}\right.
$$

for any integer $k \geq k_{0}$. Then $H^{i}\left(X, \mathcal{O}_{X}(k)\right)=0$ for $k \geq k_{0}-1$. In particular, if $\operatorname{reg}(Y) \leq k$, then $\operatorname{reg}\left(\mathcal{O}_{X}\right) \leq k-1$.

Proof. From the exact sequence

$$
0 \longrightarrow \mathcal{O}_{X}(-1) \longrightarrow \mathcal{O}_{X} \longrightarrow \mathcal{O}_{Y} \longrightarrow 0
$$

we obtain an exact sequence

$$
\cdots \rightarrow H^{i-1}\left(X, \mathcal{O}_{X}(k)\right) \rightarrow H^{i-1}\left(Y, \mathcal{O}_{Y}(k)\right) \rightarrow H^{i}\left(X, \mathcal{O}_{X}(k-1)\right) \rightarrow H^{i}\left(X, \mathcal{O}_{X}(k)\right) \rightarrow H^{i}\left(Y, \mathcal{O}_{Y}(k)\right) \rightarrow \cdots
$$

If $Y \subseteq \mathbb{P}^{r-1}$ is $k$-normal, then $H^{0}\left(X, \mathcal{O}_{X}(k)\right) \rightarrow H^{0}\left(Y, \mathcal{O}_{Y}(k)\right)$ is surjective. By the assumption in the lemma, we have

$$
H^{i}\left(X, \mathcal{O}_{X}(k-1)\right)=H^{i}\left(X, \mathcal{O}_{X}(k)\right) \text { for } k \geq k_{0} .
$$

By Serre vanishing theorem, $H^{i}\left(X, \mathcal{O}_{X}(k)\right)=0$ for $k \gg 0$. Hence the assertion follows. 
By [GLP] and [L1], the regularity conjecture holds for integral curves and smooth surfaces. Lemma 2.1 then implies that if $X \subseteq \mathbb{P}^{r}$ is a non-degenerate projective variety of dimension $n \geq 2$, codimension $e$, and degree $d$, then we have

$$
H^{n-1}\left(X, \mathcal{O}_{X}(k-n+1)\right)=H^{n}\left(X, \mathcal{O}_{X}(k-n)\right)=0 \text { for } k \geq d-e .
$$

If furthermore $n \geq 3$ and the singular locus of $X$ has codimension $\geq 3$ in $X$, then

$$
H^{n-2}\left(X, \mathcal{O}_{X}(k-n+2)\right)=0 \text { for } k \geq d-e .
$$

2.2. Castelnuovo's genus bound. The following classical result plays an important role in proving Theorem B.

Theorem 2.2 (Castelnuovo). Let $C \subseteq \mathbb{P}^{r}$ be a non-degenerate projective curve of degree $d$ and arithmetic genus $g$, and set $m:=\left\lfloor\frac{d-1}{r-1}\right\rfloor$ and $\epsilon:=(d-1)-m(r-1)$. Then we have

$$
g \leq\left(\begin{array}{c}
m \\
2
\end{array}\right)(r-1)+m \epsilon=\frac{(d-1-\epsilon)(d+\epsilon-r)}{2(r-1)} .
$$

If the equality holds, then $C \subseteq \mathbb{P}^{r}$ is projectively normal.

Corollary 2.3. Let $C \subseteq \mathbb{P}^{r}$ be a non-degenerate smooth projective curve of degree $d$ and codimension e. Then $\operatorname{reg}\left(\mathcal{O}_{C}\right) \leq\left\lfloor\frac{d-1}{e}\right\rfloor+1$.

Proof. Let $g$ be the genus of $C$, and set $m:=\left\lfloor\frac{d-1}{e}\right\rfloor$ and $\epsilon:=(d-1)-m e$. By applying Theorem 2.2, we see that $2 g-2 \leq m(m e-e+2 \epsilon)-2<m(m e+\epsilon+1)=m d$. This implies that $H^{1}\left(C, \mathcal{O}_{C}(m)\right)=0$. Thus $\operatorname{reg}\left(\mathcal{O}_{C}\right) \leq m+1$.

2.3. Roth varieties. We recall Ilic's construction of Roth varieties in [Il, Theorem 3.7]. Let $S:=\mathbb{P}\left(\mathcal{O}_{\mathbb{P}^{1}}^{\oplus 2} \oplus \mathcal{O}_{\mathbb{P}^{1}}\left(a_{1}\right) \oplus \cdots \oplus \mathcal{O}_{\mathbb{P}^{1}}\left(a_{n-1}\right)\right)$ be a rational scroll for all $a_{i} \geq 1$ with the projection $\pi_{1}: S \rightarrow \mathbb{P}^{1}$. Consider the birational morphism $\pi_{2}: S \rightarrow \bar{S} \subseteq \mathbb{P}^{N}$ given by the complete linear system $\left|\mathcal{O}_{S}(1)\right|$ of the tautological line bundle, where $N=a_{1}+\cdots+a_{n-1}+n$. For any integer $b \geq 1$, consider a smooth variety $\widetilde{X} \in\left|\mathcal{O}_{S}(b) \otimes \pi_{1}^{*} \mathcal{O}_{\mathbb{P}^{1}}(1)\right|$ such that $\left.\pi_{2}\right|_{\tilde{X}}: \widetilde{X} \stackrel{\sim}{\rightarrow} \pi_{2}(\tilde{X})=: X$ is an isomorphism. Then $X \subseteq \mathbb{P}^{N}$ is a non-degenerate linearly normal smooth projective variety of dimension $n$. A Roth variety $X \subseteq \mathbb{P}^{r}$ is an isomorphic projection of $X \subseteq \mathbb{P}^{N}$. Note that $\operatorname{deg}(X)=b(N-n)+1=b\left(a_{1}+\cdots+a_{n-1}\right)+1$. If $b=1$, then $X$ is a rational scroll. For more details, we refer to [Il, Section 3].

2.4. Double point divisors from outer projection. Let $X \subseteq \mathbb{P}^{r}$ be a non-degenerate smooth projective variety of dimension $n$, codimension $e$, and degree $d$, and $H$ be its hyperplane section. Take a general outer projection

$$
\pi=\pi_{\Lambda}: X \rightarrow \bar{X} \subseteq \mathbb{P}^{n+1}
$$

centered at an (e-2)-dimensional general linear subspace $\Lambda \subseteq \mathbb{P}^{r}$ with $\Lambda \cap X=\emptyset$. Note that $\operatorname{deg}(\bar{X})=d$. By the birational double point formula (see e.g., [L2, Lemma 10.2.8]), the non-isomorphic locus of $\pi$ defines an effective divisor $D_{\text {out }}(\Lambda)$ linearly equivalent to the double point divisor from outer projection

$$
D_{\text {out }}:=-K_{X}+(d-n-2) H .
$$

Lemma 2.4. $-K_{X}+(d-n-2) H=0$ if and only if $X \subseteq \mathbb{P}^{r}$ is a hypersurface.

Proof. The 'if' direction is trivial. For the converse, we assume that $K_{X}=(d-n-2) H$. Let $C \subseteq \mathbb{P}^{e+1}$ be a general curve section of genus $g$. Then $K_{X}=(d-3) H$ so that $2 g-2=d(d-3)$. However, if $e \geq 2$, then Theorem 2.2 implies that $2 g-2<d(d-3)$. Thus $e=1$. 
The global section $s(\Lambda) \in H^{0}\left(X, \mathcal{O}_{X}\left(D_{\text {out }}\right)\right)$ with $\operatorname{div}(s(\Lambda))=D_{\text {out }}(\Lambda)$ is called a geometric section, and the the effective divisor $D_{\text {out }}(\Lambda)$ is called a geometric divisor. Let $V_{\text {out }}$ be the linear subspace in $H^{0}\left(X, \mathcal{O}_{X}\left(D_{\text {out }}\right)\right)$ spanned by geometric sections. By varying centers of projections, Mumford proved the following.

Proposition 2.5 ([BM, Technical appendix 4]). $V_{\text {out }}$ is base point free. In particular, $D_{\text {out }}$ is base point free.

2.5. Double point divisors from inner projection. Let $X \subseteq \mathbb{P}^{r}$ be a non-degenerate smooth projective variety of dimension $n \geq 2$, codimension $e \geq 2$, and degree $d$, and $H$ be a general hyperplane section. Take a general inner projection

$$
\pi_{\Lambda}: X \rightarrow \bar{X} \subseteq \mathbb{P}^{n+1}
$$

centered at $e-1$ general points $x_{1}, \ldots, x_{e-1}$ on $X$. Note that $\operatorname{deg}(\bar{X})=d-e+1$. Let $\sigma: \widetilde{X} \rightarrow X$ be the blow-up at $x_{1}, \ldots, x_{e-1}$. We have a birational morphism $\tilde{\pi}: \widetilde{X} \rightarrow \bar{X}$. We obtain the following commutative diagram

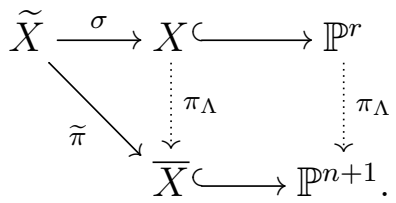

Let $\Lambda=\left\langle x_{1}, \ldots, x_{e-1}\right\rangle$ be the linear span by $x_{1}, \ldots, x_{e-1}$ so that $\Lambda$ is an $(e-2)$-dimensional linear subspace of $\mathbb{P}^{r}$.

In [No2], Noma proves that the birational morphism $\tilde{\pi}: \tilde{X} \rightarrow X$ contracts some divisors if and only if $X \subseteq \mathbb{P}^{r}$ is a scroll over a smooth projective curve or the second Veronese surface $v_{2}\left(\mathbb{P}^{2}\right) \subseteq \mathbb{P}^{5}$ (see $[$ No2, Theorem 3$\left.]\right)$. Suppose now that $X \subseteq \mathbb{P}^{r}$ is neither a scroll over a smooth projective curve nor the second Veronese surface. By the birational double point formula ([L2, Lemma 10.2.8]), the non-isomorphic locus of $\widetilde{\pi}$ defines an effective divisor $\widetilde{D}(\Lambda)$ linearly equivalent to $-K_{\widetilde{X}}+\widetilde{\pi}^{*} K_{\bar{X}}$. We define an effective divisor $D_{i n n}(\Lambda):=\sigma_{*} \widetilde{D}(\Lambda)$ on $X$. Then $D_{\text {inn }}(\Lambda)$ is linearly equivalent to the double point divisor from inner projection

$$
D_{i n n}:=-K_{X}+(d-n-e-1) H .
$$

Lemma 2.6. $-K_{X}+(d-n-e-1) H=0$ if and only if $X \subseteq \mathbb{P}^{r}$ is a linearly normal del Pezzo manifold.

Proof. The 'if' direction is trivial. For the converse, we assume that $K_{X}=(d-n-e-1) H$. Let $C \subseteq \mathbb{P}^{e+1}$ be a general curve section of genus $g$. Then $K_{C}=(d-e-2) H$ so that $2 g-2=$ $d(d-e-2)$. However, if $d \geq e+3$, then Theorem 2.2 implies that $2 g-2<d(d-e-2)$. Thus $d \leq e+2$. By considering the classification of varieties of minimal and almost minimal degree (see Remark 3.8), we can conclude that $X \subseteq \mathbb{P}^{r}$ is a linearly normal del Pezzo manifold.

The global section $s(\Lambda) \in H^{0}\left(X, \mathcal{O}_{X}\left(D_{i n n}\right)\right)$ with $\operatorname{div}(s(\Lambda))=D_{i n n}(\Lambda)$ is called a geometric section, and the the effective divisor $D_{i n n}(\Lambda)$ is called a geometric divisor. Let $V_{i n n}$ be the linear subspace in $H^{0}\left(X, \mathcal{O}_{X}\left(D_{i n n}^{\prime}\right)\right)$ spanned by geometric sections. By varying the centers of projections, Noma shows that the base locus $\operatorname{Bs}\left(\left|V_{i n n}\right|\right)$ of $V_{i n n}$ lies in the set of non-birational centers of simple inner projections (see [No2, Theorem 1]). Thus we have

$$
\operatorname{Bs}\left(\left|V_{i n n}\right|\right) \subseteq \mathcal{C}(X):=\{u \in X \mid \ell(X \cap\langle u, x\rangle) \geq 3 \text { for a general point } x \in X\} .
$$

By [No2, Corollary 6.2], we know that if $\operatorname{dim} \mathcal{C}(X) \geq 1$, then $X$ is a Roth variety. 
Theorem 2.7 ([No2, Theorem 4]). Suppose that $X \subseteq \mathbb{P}^{r}$ is neither a scroll over a smooth projective curve, the second Veronese surface, nor a Roth variety. Then $D_{i n n}$ is semiample.

\section{A sharp Castelnuovo-Mumford Regularity bound For the Structure sheaF}

This section is devoted to the proofs of Theorems A and B. Recall that Theorem A is equivalent to Theorem B $(a)$. Thus we only focus on Theorem B. We start by recalling Noma's result.

Proposition 3.1 ([No2, Corollary 5]). Let $X \subseteq \mathbb{P}^{r}$ be a non-degenerate smooth projective variety of dimension $n \geq 2$, codimension $e \geq 2$, and degree $d$. Suppose that $X \subseteq \mathbb{P}^{r}$ is neither a scroll over a smooth projective curve, the second Veronese surface, nor a Roth variety. Then we have $\operatorname{reg}\left(\mathcal{O}_{X}\right) \leq d-e$.

Proof. We include the proof for reader's convenience. By Theorem 2.7, we know that $D_{i n n}=$ $-K_{X}+(d-n-e-1) H$ is semiample so that $-K_{X}+(d-n-e-1) H+(n+1-i) H$ is ample for $1 \leq i \leq n$. It follows from Kodaira vanishing theorem that

$$
H^{i}\left(X, \mathcal{O}_{X}(d-e-i)\right)=H^{i}\left(X, \mathcal{O}_{X}\left(K_{X}+\left(-K_{X}+(d-n-e-1) H+(n+1-i) H\right)\right)\right)=0
$$

for $1 \leq i \leq n$. Thus the assertion holds.

If $X=v_{2}\left(\mathbb{P}^{2}\right) \subseteq \mathbb{P}^{5}$ is the second Veronese surface, then $\operatorname{reg}\left(\mathcal{O}_{X}\right)=1=\operatorname{deg}(X)-\operatorname{codim}(X)$. When $X \subseteq \mathbb{P}^{r}$ is a Roth variety, we can also compute $\operatorname{reg}\left(\mathcal{O}_{X}\right)$.

Proposition 3.2. Let $X \subseteq \mathbb{P}^{r}$ be a non-degenerate Roth variety of dimension $n$, codimension $e \geq 2$, and degree $d=b(N-n)+1$ for some integer $b \geq 2$, where it is an isomorphic projection of the linearly normal Roth variety $X \subseteq \mathbb{P}^{N}$. Then we have $\operatorname{reg}\left(\mathcal{O}_{X}\right)=b$. Furthermore, $\operatorname{reg}\left(\mathcal{O}_{X}\right) \leq d-e-1$ and the equality holds if and only if $b=2, n=3, e=2, d=5$.

Proof. The first assertion is shown in [No2, Corollary 5], but we include the proof for reader's convenience. We use the notations in Subsection 2.3. Note that $H^{i}\left(X, \mathcal{O}_{X}(k)\right)=0$ for $0<i<n$ and $k \in \mathbb{Z}$ (see [Il, Theorem 3.14]). Let $H$ be a general hyperplane section of $X \subseteq \mathbb{P}^{r}$, and $F$ be the restriction of a fiber of $\pi: S \rightarrow \mathbb{P}^{1}$ to $X$. Then $K_{X}=(b-n-1) H+(N-n-1) F$. By Serre duality, we have

$$
\begin{aligned}
& H^{n}\left(X, \mathcal{O}_{X}(b-1-n)\right)=H^{0}\left(X, \mathcal{O}_{X}\left(K_{X}-(b-1-n) H\right)\right)^{*}=H^{0}\left(X, \mathcal{O}_{X}((N-n-1) F)\right)^{*}, \\
& H^{n}\left(X, \mathcal{O}_{X}(b-n)\right)=H^{0}\left(X, \mathcal{O}_{X}\left(K_{X}-(b-n) H\right)\right)^{*}=H^{0}\left(X, \mathcal{O}_{X}(-H+(N-n-1) F)\right)^{*} .
\end{aligned}
$$

Now, $(N-n-1) F$ is an effective divisor, and $-H+(N-n-1) F$ is not a pseudoeffective divisor. Thus we obtain $H^{0}\left(X, \mathcal{O}_{X}((N-n-1) F)\right) \neq 0$ and $H^{0}\left(\mathcal{O}_{X}(-H+(N-n-1) F)\right)=0$. This proves that $\operatorname{reg}\left(\mathcal{O}_{X}\right)=b$. For the second assertion, suppose that $b \geq d-e-1$. Then we have

$$
b \geq d-e-1 \geq b(N-n)-e \geq b(N-n)-(N-n),
$$

so we obtain $1 \geq(b-1)(N-n-1)$. Thus $b=N-n=2$ so that $d=5, e=2$. Now, since $a_{1}+\cdots+a_{n-1}=2$, it follows that $n=3$. This completes the proof.

In view of Proposition 3.1, it only remains to consider the scroll case. To motivate our approach, we first give the proof of Theorem B for the curve case.

Proposition 3.3. Let $C \subseteq \mathbb{P}^{r}$ be a non-degenerate smooth projective curve of degree $d$, codimension $e$, and genus $g$ Then we have the following:

(1) $\operatorname{reg}\left(\mathcal{O}_{C}\right) \leq d-e$. 
(2) $\operatorname{reg}\left(\mathcal{O}_{C}\right)=d-e$ if and only if $C \subseteq \mathbb{P}^{r}$ is a plane curve, a rational normal curve $(d=e+1)$, or an elliptic normal curve $(d=e+2)$.

(3) $\operatorname{reg}\left(\mathcal{O}_{C}\right)=d-e-1$ if and only if $C \subseteq \mathbb{P}^{r}$ is an isomorphic projection of a projective variety in (2) at one point, a linearly normal curve of genus $g=2$ and degree $d=e+3$, or a complete intersection of type $(2,3)$.

Proof. Since the assertion is obvious for $e=1$, we assume that $e \geq 2$. Suppose that $d \leq e+3$. By applying Theorem 2.2, we see that $g \leq 2$. Note that $\operatorname{reg}\left(\mathcal{O}_{C}\right)=1$ if $g=0$ and $\operatorname{reg}\left(\mathcal{O}_{C}\right)=2$ if $g=1,2$. It is then easy to check the proposition. Now, suppose that $d \geq e+4$. We only have to show that $H^{1}\left(C, \mathcal{O}_{C}(d-e-3)\right)=0$ except when $C \subseteq \mathbb{P}^{r}$ is a complete intersection of type $(2,3)$. It follows from Theorem 2.2 and direct calculations that

$$
(d-e-3) d>2 g-2
$$

except when $(d, e)=(6,2)$. If $(d, e) \neq(6,2)$, then we obtain $H^{1}\left(C, \mathcal{O}_{C}(d-e-3)\right)=0$. If $(d, e)=(6,2)$ and $2 g-2 \geq(d-e-3) d=6$, then $g \geq 4$. We can easily check that the space curve of degree 6 and genus $g \geq 4$ is a complete intersection of type $(2,3)$.

Recall that a scroll $X \subseteq \mathbb{P}^{r}$ over a smooth projective curve $C$ is an isomorphic projection of $X=\mathbb{P}(E) \subseteq \mathbb{P}^{N}$, where $E$ is a very ample vector bundle on $C$ and the embedding $\mathbb{P}(E) \subseteq \mathbb{P}^{N}$ is given by the complete linear system $\left|\mathcal{O}_{\mathbb{P}(E)}(1)\right|$.

Let $E$ be a vector bundle on a smooth projective curve $C$ of genus $g$. We define

$$
\mu^{-}(E):=\min \left\{\mu(Q)=\frac{\operatorname{deg} Q}{\operatorname{rank} Q} \mid Q \text { is a quotient bundle of } E\right\} .
$$

If $E$ is semistable, then $\mu^{-}(E)=\frac{\operatorname{deg} E}{\operatorname{rank} E}$. If $E$ is not semistable, then there is a semistable quotient bundle $Q$ of $E$ with $\mu^{-}(E)=\mu(Q)=\frac{\operatorname{deg} Q}{\operatorname{rank} Q}$.

Lemma 3.4 ([B, Lemmas 1.12 and 2.5]). We have the following:

(1) $\mu^{-}\left(S^{k}(E)\right)=k \mu^{-}(E)$ for any integer $k>0$.

(2) If $\mu^{-}(E)>2 g-2$, then $H^{1}(C, E)=0$.

Lemma 3.5. Let $X \subseteq \mathbb{P}^{r}$ be a non-degenerate scroll of degree $d$ and codimension e over a smooth projective curve of genus $g$. Suppose that $n=\operatorname{dim}(X) \geq 2$. Then we have the following:

(1) If $g=0$, then $\operatorname{reg}\left(\mathcal{O}_{X}\right)=1$.

(2) If $g=1$, then $\operatorname{reg}\left(\mathcal{O}_{X}\right)=2$.

(3) If $g \geq 2$, then $\operatorname{reg}\left(\mathcal{O}_{X}\right) \leq d-e-2$.

Proof. Let $E$ be a very ample vector bundle on a smooth projective curve $C$ of genus $g$ such that $X=\mathbb{P}(E) \subseteq \mathbb{P}^{r}$ is a scroll over $C$, and $F$ be a general fiber of the natural projection $\pi: \mathbb{P}(E) \rightarrow C$. Note that $H^{i}\left(X, \mathcal{O}_{X}(k)\right)=0$ for $1<i<n$ and $k \in \mathbb{Z}$. We also have $H^{n}\left(X, \mathcal{O}_{X}(k)\right)=0$ for $k>-n$ and $H^{n}\left(X, \mathcal{O}_{X}(k)\right) \neq 0$ for $k \leq-n$. Thus we only have to consider the vanishing for $H^{1}\left(X, \mathcal{O}_{X}(k)\right)$.

If $g=0$, then $H^{1}\left(X, \mathcal{O}_{X}\right)=0$, which implies (1). If $g=1$, then $\mu^{-}(E)>0=2 g-2$ due to the very ampleness of $E$. By Lemma 3.4, $H^{1}\left(X, \mathcal{O}_{X}(1)\right)=H^{1}(C, E)=0$, which implies (2).

It only remains to consider the case that $g \geq 2$. It suffices to show that

$$
H^{1}\left(X, \mathcal{O}_{X}(d-e-3)\right)=H^{1}\left(C, S^{d-e-3}(E)\right)=0 .
$$

By Lemma 3.4, it is enough to show that

$$
(d-e-3) \mu^{-}(E)=\mu^{-}\left(S^{d-e-3}(E)\right)>2 g-2 .
$$


We consider the embedding $C \subseteq \mathbb{P}^{N}$ given by $|\operatorname{det} E|$. The main theorem of [IT] asserts that

$$
N+1=h^{0}(C, \operatorname{det} E) \geq h^{0}(C, E)+n-2 .
$$

Since $h^{0}(C, E) \geq r+1$, it follows that $N \geq r+n-2 \geq n+1$. Consider an inner projection of $C \subseteq \mathbb{P}^{N}$ centered at $N-n-1$ general points to a projective curve $\bar{C} \subseteq \mathbb{P}^{n+1}$. Note that $n+1 \geq 3$. Then we have

$$
\bar{d}:=\operatorname{deg}(\bar{C})=d-N+n+1 \leq d-(r+n-2)+n+1=d-r+3 \leq d-e+1 .
$$

Since the inner projection map $C \rightarrow \bar{C}$ is birational, the genus $g$ of $C$ is less than or equal to the arithmetic genus of $\bar{C}$. By applying Theorem 2.2 to $\bar{C} \subseteq \mathbb{P}^{n+1}$, we obtain

$$
\frac{(d-e-\epsilon)(d+\epsilon-e-n)}{n}-2 \geq \frac{(\bar{d}-1-\epsilon)(\bar{d}+\epsilon-n-1)}{n}-2 \geq 2 g-2,
$$

where $\epsilon=\bar{d}-\left\lfloor\frac{\bar{d}-1}{n}\right\rfloor n-1$. Note that $0 \leq \epsilon \leq n-1$.

First, suppose that $E$ is semistable so that $\mu^{-}(E)=\mu(E)=\frac{d}{n}$. It is easy to check that

$$
\frac{(d-e-3) d}{n}>\frac{(d-e-\epsilon)(d+\epsilon-e-n)}{n}
$$

according to $\epsilon=0,1,2$, and $\epsilon \geq 3$. By (3.2), we obtain

$$
(d-e-2) \mu^{-}(E)=\frac{(d-e-2) d}{n}>2 g-2 .
$$

Thus we verify (3.1) in the case that $E$ is semistable.

Now, suppose that $E$ is not semistable so that there is a semistable quotient bundle $Q$ of $E$ with $\mu^{-}(E)=\mu(Q)$. Note that $Q$ is also very ample. Let $d^{\prime}:=\operatorname{deg} Q$ and $n^{\prime}=\operatorname{rank} Q$. Consider the scroll $X^{\prime}=\mathbb{P}(Q) \subseteq \mathbb{P}^{n^{\prime}+e^{\prime}}$, where the embedding is given by $\left|\mathcal{O}_{\mathbb{P}(Q)}(1)\right|$. We further divide into two cases: (i) $d-e-3 \geq d^{\prime}-e^{\prime}-3$ and (ii) $d-e-3<d^{\prime}-e^{\prime}-3$. Suppose that we are in Case (i). Since $Q$ is semistable, we know that $\left(d^{\prime}-e^{\prime}-3\right) \mu(Q)>2 g-2$. Thus we have

$$
(d-e-3) \mu^{-}(E) \geq\left(d^{\prime}-e^{\prime}-3\right) \mu(Q)>2 g-2,
$$

which verifies (3.1). Suppose that we are in Case (ii). We have $d^{\prime}>d-e+e^{\prime}$ and $n>n^{\prime}$, so we obtain

$$
(d-e-3) \mu^{-}(E)=(d-e-3) \frac{d^{\prime}}{n^{\prime}}>(d-e-3) \frac{\left(d-e+e^{\prime}\right)}{n} .
$$

It is straightforward to check

$$
\frac{(d-e-3)\left(d-e+e^{\prime}\right)}{n} \geq \frac{(d-e-\epsilon)(d+\epsilon-e-n)}{n}-2
$$

according to $\epsilon=0,1,2$, and $\epsilon \geq 3$. Now (3.2) implies that

$$
(d-e-3) \mu^{-}(E)>2 g-2,
$$

which verifies (3.1). Therefore, we complete the proof.

Proposition 3.6. Let $X \subseteq \mathbb{P}^{r}$ be a non-degenerate scroll of degree $d$ and codimension e over a smooth projective curve of genus $g$. Suppose that $n=\operatorname{dim}(X) \geq 2$. Then we have the following:

(1) $\operatorname{reg}\left(\mathcal{O}_{X}\right) \leq d-e$.

(2) $\operatorname{reg}\left(\mathcal{O}_{X}\right)=d-e$ if and only if $X \subseteq \mathbb{P}^{r}$ is a rational normal scroll $(d=e+1)$.

(3) $\operatorname{reg}\left(\mathcal{O}_{X}\right)=d-e-1$ if and only if $X \subseteq \mathbb{P}^{r}$ is an isomorphic projection of (2) at one point or an elliptic normal surface scroll with $d=e+3$. 
Proof. By Lemma 3.5, we only have to consider the case that $g \leq 1$. If $g=0$, then $\operatorname{reg}\left(\mathcal{O}_{X}\right)=$ $1 \leq d-e$ and the equality holds if and only if $X \subseteq \mathbb{P}^{r}$ is a rational normal scroll. Suppose now that $g=1$. Then $d \geq r+1=n+e+1$, and the equality holds only if $X \subseteq \mathbb{P}^{r}$ is linearly normal by $\left[\mathrm{KP}\right.$, Theorem 1.1]. We have $\operatorname{reg}\left(\mathcal{O}_{X}\right)=2 \leq n \leq d-e-1$ and the equality holds if and only if $n=2, d=e+3$, i.e., $X \subseteq \mathbb{P}^{r}$ is an elliptic normal scroll with $d=e+3$. This completes the proof.

Remark 3.7. Let $X=\mathbb{P}(E) \subseteq \mathbb{P}^{r}$ be a non-degenerate scroll of degree $d$ and codimension $e$ over a smooth projective curve $C$ of genus $g$, and $F$ be a general fiber of the natural projection $\pi: X \rightarrow C$. Consider the divisor $D:=-K_{X}+(d-n-e-1) H \equiv(d-e-1) H-(d+2 g-2) F$. If $D$ is nef, then Kodaira vanishing theorem implies that $\operatorname{reg}\left(\mathcal{O}_{X}\right) \leq d-n-e-1$, which is stronger than Lemma 3.5. By Miyaoka's criterion (see [B, Lemma 5.4]), $D$ is nef if and only if $(d-e-1) \mu^{-}(E) \geq d+2 g-2$. The similar argument in the proof of Lemma 3.5 shows that $D$ is nef when $E$ is semistable. However, we do not know whether $D$ is nef in general.

We are ready to prove Theorem $B$.

Proof of Theorem $B$. Let $X \subseteq \mathbb{P}^{r}$ be a non-degenerate smooth projective variety of dimension $n$, codimension $e$, and degree $d$, and $H$ be its hyperplane section. If $X \subseteq \mathbb{P}^{r}$ is a hypersurface, then the assertion is trivial. We already show the assertion for the curve case in Proposition 3.3. Thus we assume that $e \geq 2$ and $n \geq 2$.

If $X \subseteq \mathbb{P}^{r}$ is the second Veronese surface $v_{2}\left(\mathbb{P}^{2}\right) \subseteq \mathbb{P}^{5}$, then $d=e+1$ and $\operatorname{reg}\left(\mathcal{O}_{X}\right)=1=d-e$. If $X \subseteq \mathbb{P}^{r}$ is a Roth variety or a scroll over a smooth projective curve, then the assertion follows from Propositions 3.2 and 3.6, respectively. Thus we further assume that $X \subseteq \mathbb{P}^{r}$ is neither the second Veronese surface, a Roth variety, nor a scroll over a smooth projective curve.

(a) The assertion follows from Proposition 3.1.

(b) The 'if' part is trivial by the classification of varieties with $d \leq e+2$ (see Remark 3.8). For the 'only if' part, we assume that $\operatorname{reg}\left(\mathcal{O}_{X}\right)=d-e$, i.e., $\mathcal{O}_{X}$ fails to be $(d-e-1)$-regular. In this case, we observe that $X \subseteq \mathbb{P}^{r}$ is linearly normal. To see this, suppose that $X \subseteq \mathbb{P}^{r}$ is not linearly normal. Then it is an isomorphic projection of $X \subseteq \mathbb{P}^{r+1}$, which has degree $d$ and codimension $e+1$. By (a) for $X \subseteq \mathbb{P}^{r+1}$, we have $\operatorname{reg}\left(\mathcal{O}_{X}\right) \leq d-(e+1)=d-e-1$, so we get a contradiction.

By Theorem 2.7, $D_{i n n}=-K_{X}+(d-n-e-1) H$ is semiample. Then the divisor

$$
-K_{X}+(d-n-e-1) H+(n-i) H=-K_{X}+(d-e-1-i) H
$$

is ample for $1 \leq i \leq n$. It follows from Kodaira vanishing theorem that

$$
H^{i}\left(X, \mathcal{O}_{X}(d-e-1-i)\right)=0 \text { for } 1 \leq i \leq n-1 .
$$

Thus we must have

$$
H^{n}\left(X, \mathcal{O}_{X}(d-e-1-n)\right) \neq 0 .
$$

Let $Y \subseteq \mathbb{P}^{r-1}$ be a smooth general hyperplane section of $X \subseteq \mathbb{P}^{r}$. Consider the following exact sequence

$$
0 \longrightarrow \mathcal{O}_{X}(d-e-n-1) \longrightarrow \mathcal{O}_{X}(d-e-n) \longrightarrow \mathcal{O}_{Y}(d-e-n) \longrightarrow 0 .
$$

Since $\mathcal{O}_{X}$ is $(d-e)$-regular, we have $H^{n}\left(X, \mathcal{O}_{X}(d-e-n)\right)=0$. Thus the map

$$
H^{n-1}\left(Y, \mathcal{O}_{Y}(d-e-1-(n-1))\right) \rightarrow H^{n}\left(X, \mathcal{O}_{X}(d-e-n-1)\right)
$$

is surjective, so we obtain $H^{n-1}\left(Y, \mathcal{O}_{Y}(d-e-1-(n-1))\right) \neq 0$. Consequently, for a general smooth curve section $C \subseteq \mathbb{P}^{e+1}$ of $X \subseteq \mathbb{P}^{r}$, we can conclude that $H^{1}\left(C, \mathcal{O}_{C}(d-e-2)\right) \neq 0$. By 
Proposition 3.3, $C \subseteq \mathbb{P}^{e+1}$ is either a rational normal curve or an elliptic normal curve. Thus $d \leq e+2$, so we complete the proof for $(b)$.

(c) The 'if' part is trivial by the classification result in Remark 3.8. For the 'only' if part, we assume that $\operatorname{reg}\left(\mathcal{O}_{X}\right)=d-e-1$. In particular, $\mathcal{O}_{X}$ fails to be $(d-e-2)$-regular. If $X \subseteq \mathbb{P}^{r}$ is not linearly normal, then it is an isomorphic projection of a projective variety $X \subseteq \mathbb{P}^{r+1}$ of degree $d$ and codimension $e+1$. Since $\operatorname{reg}\left(\mathcal{O}_{X}\right)=d-(e+1)$, it follows that $X \subseteq \mathbb{P}^{r+1}$ is a projective variety in (b). Thus we may assume that $X \subseteq \mathbb{P}^{r}$ is linearly normal.

By Theorem 2.7, $D_{i n n}=-K_{X}+(d-n-e-1) H$ is semiample. Then the divisor

$$
-K_{X}+(d-n-e-1) H+(n-1-i) H=-K_{X}+(d-e-2-i) H
$$

is ample for $1 \leq i \leq n-2$. It follows from Kodaira vanishing theorem that

$$
H^{i}\left(X, \mathcal{O}_{X}(d-e-2-i)\right)=0 \text { for } 1 \leq i \leq n-2 .
$$

Thus we have

$$
H^{n}\left(X, \mathcal{O}_{X}(d-e-2-n)\right) \neq 0 \text { or } H^{n-1}\left(X, \mathcal{O}_{X}(d-e-1-n)\right) \neq 0 .
$$

Note that $\operatorname{reg}\left(\mathcal{O}_{X}\right)=d-e-1$ implies that

$$
H^{n}\left(X, \mathcal{O}_{X}(d-e-1-n)\right)=0 \text { and } H^{n-1}\left(X, \mathcal{O}_{X}(d-e-n)\right)=0 .
$$

Let $Y \subseteq \mathbb{P}^{r-1}$ be a smooth general hyperplane section of $X \subseteq \mathbb{P}^{r}$. If $H^{n}\left(X, \mathcal{O}_{X}(d-e-2-n)\right) \neq$ 0 , then by considering the following short exact sequence

$$
0 \longrightarrow \mathcal{O}_{X}(d-e-2-n) \longrightarrow \mathcal{O}_{X}(d-e-1-n) \longrightarrow \mathcal{O}_{Y}(d-e-1-n) \longrightarrow 0,
$$

we see that $H^{n-1}\left(Y, \mathcal{O}_{Y}(d-e-2-(n-1))\right) \neq 0$. Similarly, one can also check that if $H^{n-1}\left(X, \mathcal{O}_{X}(d-e-1-n)\right) \neq 0$, then $H^{n-2}\left(Y, \mathcal{O}_{Y}(d-e-1-(n-1))\right) \neq 0$. Consequently, for a general smooth surface section $S \subseteq \mathbb{P}^{e+2}$ of $X \subseteq \mathbb{P}^{r}$, we have

$$
H^{2}\left(S, \mathcal{O}_{S}(d-e-4)\right) \neq 0 \text { or } H^{1}\left(S, \mathcal{O}_{S}(d-e-3)\right) \neq 0 .
$$

Note that $H^{2}\left(S, \mathcal{O}_{S}(d-e-3)=0\right.$ and $H^{1}\left(S, \mathcal{O}_{S}(d-e-2)=0\right.$.

We now claim that

$$
H^{2}\left(S, \mathcal{O}_{S}(d-e-4)\right) \neq 0 \text { and } H^{1}\left(S, \mathcal{O}_{S}(d-e-3)\right)=0 .
$$

To show the claim, it is sufficient to prove that $H^{1}\left(S, \mathcal{O}_{S}(d-e-3)\right)=0$. By abuse of notation, we denote by $H$ a general hyperplane section of $S \subseteq \mathbb{P}^{e+2}$. Since $X \subseteq \mathbb{P}^{r}$ is neither a second Veronese surface nor a scroll over a curve, so is $S \subseteq \mathbb{P}^{e+2}$. If $S \subseteq \mathbb{P}^{e+2}$ is a Roth variety, then $H^{1}\left(S, \mathcal{O}_{S}(k)\right)=0$ for all $k \in \mathbb{Z}$. Thus, by Theorem 2.7 , we may assume that $D_{\text {inn }}=-K_{S}+(d-e-3) H$ is semiample.

We now show that $D_{i n n}$ is big possibly except one case. Consider the divisors $D:=(d-e-2) H$ and $E:=K_{S}+H$ on $S$. By [Io, Theorem 1.4], if $E$ is not base point free, then $S$ is a second Veronese surface, a quadric hypersurface, or a scroll over a curve. Those cases are already excluded, so we may assume that $E$ is base point free. Let $C \subseteq \mathbb{P}^{e+1}$ be a general smooth curve section of $S \subseteq \mathbb{P}^{e+2}$, and $g$ be the genus of $C$. We have

$D^{2}-2 D \cdot E=(d-e-2)^{2} d-2(d-e-2)\left(K_{S} \cdot H+H^{2}\right)=(d-e-2)\{(d-e-2) d-4 g+4\}$.

The following inequality

$$
\frac{(d-e-2) d+4}{4}>g,
$$


implies that $D^{2}-2 D \cdot E>0$ so that $D-E=D_{\text {inn }}$ is big by [L2, Theorem 2.2.15]. Recall from Theorem 2.2 that

$$
\frac{(d-1-\epsilon)(d+\epsilon-e-1)}{2 e} \geq g
$$

where $\epsilon=d-1-\left\lfloor\frac{d-1}{e}\right\rfloor e$ and $0 \leq \epsilon \leq e-1$. To show (3.4), it is enough to prove that

$$
\frac{(d-e-2) d+4}{4}>\frac{(d-1-\epsilon)(d+\epsilon-e-1)}{2 e},
$$

which is equivalent to

$$
(e-2)(d-e-2) d+4 e>2(e+1-\epsilon)(\epsilon+1) .
$$

We divide into two cases according to $e=2$ and $e \geq 3$. Suppose that $e \geq 3$. If $d \leq e+2$, then $H^{1}\left(S, \mathcal{O}_{S}(k)\right)=0$ for $k \in \mathbb{Z}$. Thus we can assume that $d \geq e+3$. If $\epsilon \leq 2$, then $2(e+1-\epsilon)(\epsilon+1) \leq 6 e-6$. Since we have $(e-2)(d-e-2) d>2(e-3)$, we get (3.5). If $\epsilon \geq 3$, then $d \geq e+4$. Since we have $(e-2)(d-e-2) d>(e+1-\epsilon) 2(\epsilon+1)$, we also get (3.5). Suppose now that $e=2$. When $\epsilon=0$, it is straightforward to verify (3.5). We have shown that $D_{i n n}$ is big except when $(e, \epsilon)=(2,1)$. Thus $D_{i n n}$ is now nef and big possibly except one case, and hence, it follows from Kawamata-Viehweg vanishing theorem that $H^{1}\left(S, \mathcal{O}_{S}(d-e-3)\right)=0$.

It only remains to consider the case that $(e, \epsilon)=(2,1)$. Theorem 2.2 says that $\frac{(d-2)^{2}}{4} \geq g$. If (3.4) does not hold, then we must have $\frac{(d-2)^{2}}{4}=g$. By Theorem 2.2 again, $C \subseteq \mathbb{P}^{e+1}$ is projectively normal. This implies that the natural restriction map

$$
H^{0}\left(S, \mathcal{O}_{S}(d-e-2)\right) \longrightarrow H^{0}\left(C, \mathcal{O}_{C}(d-e-2)\right)
$$

is surjective. Recall that $H^{1}\left(S, \mathcal{O}_{S}(d-e-2)\right)=0$. By considering an exact sequence

$$
0 \longrightarrow \mathcal{O}_{S}(d-e-3) \longrightarrow \mathcal{O}_{S}(d-e-2) \longrightarrow \mathcal{O}_{C}(d-e-2) \longrightarrow 0
$$

we get $H^{1}\left(S, \mathcal{O}_{S}(d-e-3)\right)=0$. This completes the proof of the claim (3.3).

We continue the proof of Theorem B $(c)$. Let $C \subseteq \mathbb{P}^{e+1}$ be a general smooth curve section of $S \subseteq \mathbb{P}^{e+2}$, and $g$ be the genus of $C$. By the claim (3.3), we see that $H^{1}\left(C, \mathcal{O}_{C}(d-e-3)\right) \neq 0$ so that $\mathcal{O}_{C}$ fails to be $(d-e-2)$-regular. By Proposition 3.3, we have either $d \leq e+3, g \leq 2$ or $e=2, d=6, g=4$. Consider the first case $(d \leq e+3, g \leq 2)$. We already assume that $X \subseteq \mathbb{P}^{r}$ is linearly normal and is not a scroll over a curve. If $g \leq 1$, then $X \subseteq \mathbb{P}^{r}$ is a variety of minimal or almost minimal degree, so $\operatorname{reg}\left(\mathcal{O}_{X}\right)=d-e \neq d-e-1$. Thus $g=2$, and consequently, $d=e+3$. In the second case $(e=2, d=6, g=4)$, we can check that $X \subseteq \mathbb{P}^{r}$ is a complete intersection of type $(2,3)$. Therefore, we complete the proof of Theorem B $(c)$.

Remark 3.8. Let $X \subseteq \mathbb{P}^{r}$ be a smooth projective variety of degree $d$ and codimension $e$. Note that $d \geq e+1$.

(1) $d=e+1: X \subseteq \mathbb{P}^{r}$ is called a variety of minimal degree. By del Pezzo-Bertini (see e.g., $[\mathrm{EH}]$ ), it is known that such a variety is either a quadric hypersurface, the second Veronese surface $v_{2}\left(\mathbb{P}^{2}\right) \subseteq \mathbb{P}^{5}$, a rational normal curve, or a rational normal scroll.

(2) $d=e+2: X \subseteq \mathbb{P}^{r}$ is called a variety of almost minimal degree. Such a variety is either an isomorphic projection from a variety of minimal degree at one point, an elliptic normal curve, or a linearly normal del Pezzo manifold. Fujita completely classified del Pezzo manifolds in [F1] and [F2].

(3) $d=e+3$ : If $X \subseteq \mathbb{P}^{r}$ is not linearly normal, then it is obtained from an isomorphic projection of a variety of degree $d \leq e+2$. Ionescu classified linearly normal varieties $X \subseteq \mathbb{P}^{r}$ of degree $d=e+3$ (see [Io, Theorem 3.12]) as follows: $X \subseteq \mathbb{P}^{r}$ is either a quartic hypersurface, an 
elliptic normal surface scroll, or a linearly normal variety with sectional genus $g=2$ which is not a scroll over a curve. The last case is either a blowing-up of Hirzebruch surfaces $F_{n}=\mathbb{P}\left(\mathcal{O}_{\mathbb{P}^{1}} \oplus \mathcal{O}_{\mathbb{P}^{1}}(-n)\right)$ with $0 \leq n \leq 2$ at $k \leq 7$ points (see [Io, Proposition 3.1]) or one of 6 cases in higher dimensions (see [Io, Theorem 3.4]).

\section{A Castelnuovo-Mumford Regularity Bound for Smooth Projective varieties}

The aim of this section is to prove Theorem $\mathrm{C}$. The following is used in $[\mathrm{C}]$ and $[\mathrm{BM}$, Technical appendix 4] (see also [P, Section II]), but we include the proof for reader's convenience.

Lemma 4.1. Let $D \in|V|$ be an effective divisor on $X$. Then the natural restriction map

$$
H^{0}\left(\mathbb{P}^{r}, \mathcal{I}_{D \mid \mathbb{P}^{r}}(d-1+\ell)\right) \longrightarrow H^{0}\left(X, \mathcal{I}_{D \mid X}(d-1+\ell)\right)
$$

is surjective for any integer $\ell$.

Proof. We first consider the case that $D=D_{\text {out }}(\Lambda)$ is a geometric divisor associated to a general outer projection $\pi=\pi_{\Lambda}: X \rightarrow \bar{X} \subseteq \mathbb{P}^{n+1}$ centered at a general linear subspace $\Lambda \subseteq \mathbb{P}^{r}$. Let $\bar{D}:=\pi(D)$ be the image of $D$ under the projection $\pi$. Then $\bar{D}$ is a Weil divisor on $\bar{X}$. We have the following diagram:

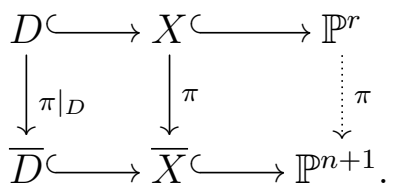

Note that $\mathcal{I}_{\bar{D} \mid \bar{X}} \simeq \pi_{*} \mathcal{I}_{D \mid X}=\pi_{*} \mathcal{O}_{X}(-D)$ is the conductor ideal sheaf of the finite birational morphism $\pi$ (see [BM, Technical appendix 4]). Consider the following short exact sequence

$$
0 \longrightarrow \mathcal{I}_{\bar{X} \mid \mathbb{P}^{n+1}} \longrightarrow \mathcal{I}_{\bar{D} \mid \mathbb{P}^{n+1}} \longrightarrow \mathcal{I}_{\bar{D} \mid \bar{X}} \longrightarrow 0
$$

We have the following commutative diagram:

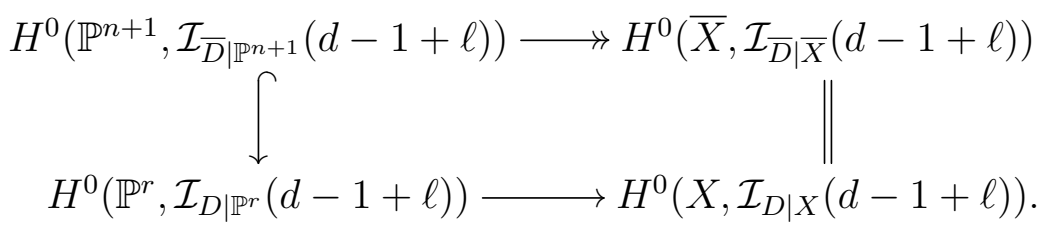

Since $\mathcal{I}_{\bar{X} \mid \mathbb{P}^{n+1}} \simeq \mathcal{O}_{\mathbb{P}^{n+1}}(-d)$ and $H^{1}\left(\mathbb{P}^{n+1}, \mathcal{O}_{\mathbb{P}^{n+1}}(\ell-1)\right)=0$, it follows that the upper horizontal map is surjective. Thus the lower horizontal map is also surjective.

Now, let $s(\Lambda) \in V$ be a geometric section associated to a general outer projection $\pi=\pi_{\Lambda}$. We may regard $H^{0}\left(X, \mathcal{I}_{D_{\text {out }}(\Lambda) \mid X}(d-1+\ell)\right)$ as a linear subspace $s(\Lambda) \cdot H^{0}\left(X, \mathcal{O}_{X}\left(K_{X}+(n+1+\ell) H\right)\right)$ of $H^{0}\left(X, \mathcal{O}_{X}(d-1+\ell)\right)$. We have shown in the previous paragraph that every global section in $s(\Lambda) \cdot H^{0}\left(X, \mathcal{O}_{X}\left(K_{X}+(n+1+\ell) H\right)\right)$ is lifted to a global section in $H^{0}\left(\mathbb{P}^{r}, \mathcal{O}_{\mathbb{P}^{r}}(d-1+\ell)\right)$. Since every global section $s \in V$ is a linear sum of geometric sections, it follows that every global section in $s \cdot H^{0}\left(X, \mathcal{O}_{X}\left(K_{X}+(n+1+\ell) H\right)\right)$ is lifted to a global section in $H^{0}\left(\mathbb{P}^{r}, \mathcal{O}_{\mathbb{P}^{r}}(d-1+\ell)\right)$. This implies the lemma.

Corollary 4.2. Let $X \subseteq \mathbb{P}^{r}$ be a smooth projective variety of degree $d$, and $D \in\left|V_{\text {out }}\right|$ be an effective divisor on $X$. Then $X \subseteq \mathbb{P}^{r}$ is $(d-1+\ell)$-normal for some integer $\ell$ if and only if the map

$$
H^{1}\left(\mathbb{P}^{r}, \mathcal{I}_{D \mid \mathbb{P}^{r}}(d-1+\ell)\right) \longrightarrow H^{1}\left(X, \mathcal{I}_{D \mid X}(d-1+\ell)\right)
$$

is injective. In particular, if $D \subseteq \mathbb{P}^{r}$ is $(d-1+\ell)$-normal, then so is $X \subseteq \mathbb{P}^{r}$. 
Proof. We consider an exact sequence

$$
0 \longrightarrow \mathcal{I}_{X \mid \mathbb{P}^{r}} \longrightarrow \mathcal{I}_{D \mid \mathbb{P}^{r}} \longrightarrow \mathcal{I}_{D \mid X} \longrightarrow 0 .
$$

By Lemma 4.1, the above exact sequence induces the following exact sequence $0 \longrightarrow H^{1}\left(\mathbb{P}^{r}, \mathcal{I}_{X \mid \mathbb{P}^{r}}(d-1+\ell)\right) \longrightarrow H^{1}\left(\mathbb{P}^{r}, \mathcal{I}_{D \mid \mathbb{P}^{r}}(d-1+\ell)\right) \longrightarrow H^{1}\left(X, \mathcal{I}_{D \mid X}(d-1+\ell)\right) \longrightarrow \cdots$

Then the assertion immediately follows.

In $[\mathrm{M}]$, Mumford proved that any smooth projective variety $X \subseteq \mathbb{P}^{r}$ of degree $d$ is schemetheoretically cut out by hypersurfaces of degree $d$ in $\mathbb{P}^{r}$. We need a generalization of this result to some singular varieties.

Lemma 4.3. Let $X \subseteq \mathbb{P}^{r}$ be a projective variety of degree d. Suppose that $X$ has only finitely many singular points and the tangent cone of each singular point is a finite union of distinct linear subspaces of $\mathbb{P}^{r}$. Then $X \subseteq \mathbb{P}^{r}$ is scheme-theoretically cut out by hypersurfaces of degree $d$ in $\mathbb{P}^{r}$.

Proof. Let $n:=\operatorname{dim}(X)$. As in $[\mathrm{M}]$, we consider a linear subspace $\Lambda$ of dimension $r-n-2$ in $\mathbb{P}^{r}$ disjoint from $X$ and the join $H_{\Lambda}$ of $X$ and $\Lambda$ (the locus of lines joining $X$ and $\Lambda$ ). Then $H_{\Lambda}$ is a hypersurface of degree $\leq d$ in $\mathbb{P}^{r}$. It is easy to see that

$$
X=\bigcap_{\Lambda \cap X=\emptyset} H_{\Lambda}
$$

as sets. By the conditions on singularities of $X$, we have

$$
T C_{p}(X)=\bigcap_{\Lambda \cap X=\emptyset} T C_{p}\left(H_{\Lambda}\right) \text { for any point } p \in X,
$$

where $T C$ stands for the tangent cone. Thus the assertion follows.

Remark 4.4. Let $X \subseteq \mathbb{P}^{r}$ be a non-degenerate smooth projective variety of dimension $n$, codimension $e \geq n+1$, and degree $d$. We take a general outer projection

$$
\pi: X \rightarrow X^{\prime} \subseteq \mathbb{P}^{2 n} .
$$

We then claim that $X^{\prime} \subseteq \mathbb{P}^{2 n}$ is $k$-normal for $k \geq n(d-2)$. This claim plays a crucial role in proving Theorem C. Notice that $X^{\prime}$ has only finitely many double points and the tangent cone of each singular point is the union of two $n$-dimensional linear subspaces of $\mathbb{P}^{2 n}$ meeting at one point (see [dFE, Example 5.7]). By Lemma 4.3, $X^{\prime} \subseteq \mathbb{P}^{2 n}$ is scheme-theoretically cut out by hypersurfaces of degree $d$ in $\mathbb{P}^{2 n}$. Since the pair $\left(\mathbb{P}^{2 n}, n X^{\prime}\right)$ is $\log$ canonical by [dFE, Example 5.7], it follows from [dFE, Corollary 5.1] that

$$
H^{1}\left(\mathbb{P}^{2 n}, \mathcal{I}_{X^{\prime} \mid \mathbb{P}^{2 n}}(k)\right)=0 \text { for } k \geq n d-2 n,
$$

which shows the desired claim.

We are ready to give the proof of Theorem C.

Proof of Theorem $C$. Recall that $X \subseteq \mathbb{P}^{r}$ is a non-degenerate smooth projective variety of dimension $n$, codimension $e \geq 2$, and degree $d$. We want to show that

$$
\operatorname{reg}(X) \leq n(d-2)+1 .
$$

By [GLP], we may assume that $n \geq 2$. It is easy to check that $d-e \leq n(d-2)$. Thus, by Theorem B, it sufficient to show that $X \subseteq \mathbb{P}^{r}$ is $n(d-2)$-normal. Suppose that $e \leq n$. It then 
follows from [No3, Theorem 9] that $X \subseteq \mathbb{P}^{r}$ is $k$-normal for any integer $k \geq e(d-e)-n$. Since $e(d-e)-n \leq n(d-2)+1$, the assertion holds for the case that $e \leq n$.

It only remains to prove the assertion for the case that $e \geq n+1$. If $e \geq n+2$, then we take a general isomorphic projection to $\mathbb{P}^{2 n+1}$. Note that if $X \subseteq \mathbb{P}^{2 n+1}$ is $n(d-2)$-normal, then so is $X \subseteq \mathbb{P}^{r}$. Thus we may assume that $e=n+1$. Take a general member $D \in\left|V_{\text {out }}\right|$. By Corollary 4.2 , it suffices to show that $D \subseteq \mathbb{P}^{2 n+1}$ is $n(d-2)$-normal. Now, take a general projection

$$
\pi: X \rightarrow X^{\prime} \subseteq \mathbb{P}^{2 n}
$$

such that $\left.\pi\right|_{D}$ is an isomorphism. It is enough to prove that $D \subseteq \mathbb{P}^{2 n}$ is $n(d-2)$-normal. Recall from Remark 4.4 that $X^{\prime} \subseteq \mathbb{P}^{2 n}$ is $n(d-2)$-normal. Thus we only have to prove that the restriction map

$$
H^{0}\left(X^{\prime}, \mathcal{O}_{X^{\prime}}(n(d-2))\right) \longrightarrow H^{0}\left(D, \mathcal{O}_{D}(n(d-2))\right)
$$

is surjective. Notice that this surjection follows from the cohomology vanishing

$$
H^{1}\left(X^{\prime}, \mathcal{I}_{D \mid X^{\prime}}(n(d-2))\right)=0,
$$

which we shall show below.

Let $Y \subseteq X^{\prime}$ be a general hyperplane section of $X^{\prime} \subseteq \mathbb{P}^{2 n}$ so that $Y \subseteq \mathbb{P}^{2 n-1}$ is a nondegenerate smooth projective variety of dimension $n-1$, codimension $n$, degree $d$. By induction on $n$, we may suppose that the theorem holds for smooth projective varieties of dimension $\leq n-1$. Thus $Y \subseteq \mathbb{P}^{2 n-1}$ is $((n-1)(d-2)+1)$-regular. By Lemma 2.1, we have

$$
H^{1}\left(X^{\prime}, \mathcal{O}_{X^{\prime}}((n-1)(d-2)-1)\right)=0 .
$$

We next consider the following commutative diagram with exact sequences

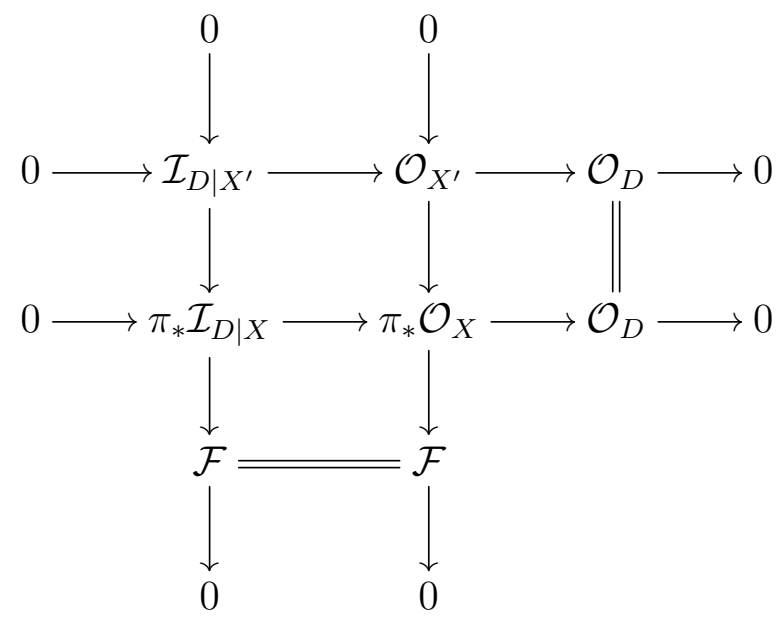

where $\mathcal{F} \simeq \bigoplus_{i} \mathcal{O}_{p_{i}}$ is a coherent sheaf supported at singular points $p_{i}$ of $X^{\prime}$. By the cohomology vanishing (4.1), the restriction map

$$
H^{0}\left(X^{\prime}, \pi_{*} \mathcal{O}_{X}((n-1)(d-2)-1)\right) \longrightarrow H^{0}\left(X^{\prime}, \mathcal{F}\right)
$$

is surjective. Recall that $D \sim-K_{X}+(d-n-2) H$, where $H$ is a hyperplane section of $X \subseteq \mathbb{P}^{2 n+1}$. Then we have $\mathcal{I}_{D \mid X}(d-1) \simeq \mathcal{O}_{X}\left(K_{X}+(n+1) H\right)$. Notice that $K_{X}+(n+1) H$ is base point free. There is a global section in $H^{0}\left(X^{\prime}, \pi_{*} \mathcal{I}_{D \mid X}(d-1)\right) \simeq H^{0}\left(X, \mathcal{O}_{X}\left(K_{X}+(n+1) H\right)\right)$ such that it does not vanish at any singular points $p_{i}$ of $X^{\prime}$. By considering the surjection of (4.2) and the multiplication map

$$
H^{0}\left(X^{\prime}, \pi_{*} \mathcal{I}_{D \mid X}(d-1)\right) \otimes H^{0}\left(X^{\prime}, \pi_{*} \mathcal{O}_{X}((n-1)(d-2)-1)\right) \longrightarrow H^{0}\left(X^{\prime}, \pi_{*} \mathcal{I}_{D \mid X}(n(d-2))\right),
$$


we see that the restriction map

$$
H^{0}\left(X^{\prime}, \pi_{*} \mathcal{I}_{D \mid X}(n(d-2))\right) \longrightarrow H^{0}\left(X^{\prime}, \mathcal{F}\right)
$$

is surjective. Since we have $H^{1}\left(X^{\prime}, \pi_{*} \mathcal{I}_{D \mid X}(n(d-2))\right)=H^{1}\left(X, \mathcal{I}_{D \mid X}(n(d-2))\right)=0$ by Kodaira vanishing theorem, it follows that

$$
H^{1}\left(X^{\prime}, \mathcal{I}_{D \mid X^{\prime}}(n(d-2))\right)=0 .
$$

This finishes the proof.

\section{A Castelnuovo-Type normality bound By double point Divisors}

In this section, we prove Theorems D and E, and discuss some related issues. We start by recalling a vanishing theorem of Ein-Lazarsfeld in [EL], which plays an important role in proving Theorems $\mathrm{D}$ and $\mathrm{E}$. Let $L$ be a globally generated line bundle on a smooth projective variety $X$, and $W \subseteq H^{0}(X, L)$ be a base point free subspace. Then we have a short exact sequence

$$
0 \longrightarrow M_{W} \longrightarrow W \otimes \mathcal{O}_{X} \stackrel{e_{W}}{\longrightarrow} L \longrightarrow 0 .
$$

If $W=H^{0}(X, L)$, we let $M_{L}:=M_{W}$. Now, let $H$ be a very ample line bundle on $X$, and $B, C$ be nef line bundles on $X$. For integers $f, g$, we write $L_{f}=K_{X}+f H+B$ and $N_{g}=K_{X}+g H+C$. Since we are working in characteristic zero, $\wedge^{q} M_{L_{f}}$ is a direct summand of $M_{L_{f}}^{\otimes q}$. Thus the original statements of [EL, Theorem 2.1 and Proposition 3.1] implies the following.

Theorem 5.1 ([EL, Theorem 2.1 and Proposition 3.1]). Assume that $(X, H) \neq\left(\mathbb{P}^{n}, \mathcal{O}_{\mathbb{P}^{n}}(1)\right)$. Then $H^{i}\left(X, \wedge^{q} M_{L_{f}} \otimes N_{g}\right)=0$ for $i \geq 1, f \geq n+1$, and $g \geq n+q-i$.

5.1. Outer projection case. Throughout this subsection, we use the following notations. Let $X \subseteq \mathbb{P}^{r}$ be a non-degenerate smooth projective variety of dimension $n$, codimension $e$, and degree $d$, and $H$ be its hyperplane section. Since the regularity conjecture holds for smooth curves and hypersurfaces, we assume that $n \geq 2$ and $e \geq 2$. Recall that

$$
D_{\text {out }}=-K_{X}+(d-n-2) H .
$$

is the double point divisor from outer projection. Let $V:=V_{\text {out }}$ be the linear subspace in $H^{0}\left(X, \mathcal{O}_{X}\left(D_{\text {out }}\right)\right)$ spanned by geometric sections. By Proposition 2.5, $V$ is a base point free. For an integer $k \geq 0$, let $V_{k}$ be the image of the multiplication map

$$
V \otimes H^{0}\left(X, \mathcal{O}_{X}(k)\right) \longrightarrow H^{0}\left(X, \mathcal{O}_{X}\left(D_{\text {out }}+k H\right)\right),
$$

and $c_{k}$ be the codimension of $V_{k}$ in $H^{0}\left(X, \mathcal{O}_{X}\left(D_{\text {out }}+k H\right)\right)$.

As a consequence of Lemma 4.1, we prove the following.

Lemma 5.2 (A generalized Mumford's lemma for outer projections). If the multiplication map

$$
V_{k} \otimes H^{0}\left(X, \mathcal{O}_{X}\left(K_{X}+(n+1+\ell) H\right)\right) \longrightarrow H^{0}\left(X, \mathcal{O}_{X}(d-1+k+\ell)\right)
$$

is surjective for some integers $k \geq 0$ and $\ell \in \mathbb{Z}$, then $X \subseteq \mathbb{P}^{r}$ is $(d-1+k+\ell)$-normal.

Proof. By considering the following commutative diagram

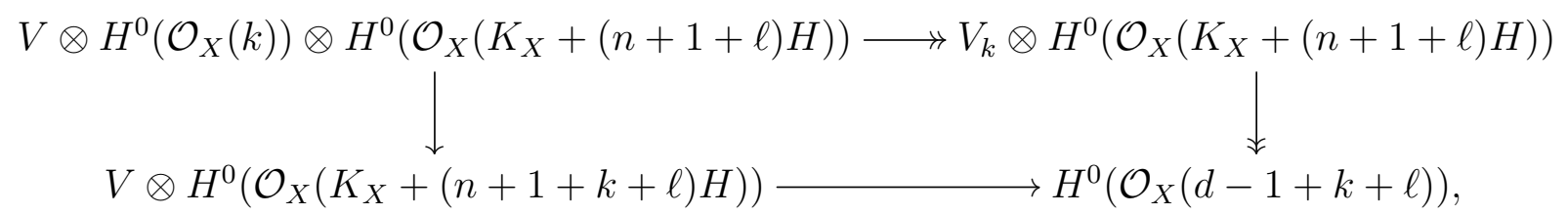


we see that the bottom map is surjective. Let $D \in|V|$ and $E \in\left|K_{X}+(n+1+k+\ell) H\right|$ be any effective divisors. Then, by Lemma 4.1, the effective divisor $D+E \in|(d-1+k+\ell) H|$ is cut out by hypersurface of degree $d-1+k+\ell$ in $\mathbb{P}^{r}$. This implies that the restriction map

$$
H^{0}\left(\mathbb{P}^{r}, \mathcal{O}_{\mathbb{P}^{r}}(d-1+k+\ell)\right) \longrightarrow H^{0}\left(X, \mathcal{O}_{X}(d-1+k+\ell)\right)
$$

is surjective.

We recall

$$
m:=\min \left\{c_{k}+k \mid-2 K_{X}+(d-2 n-3+k) H \text { is nef }\right\} .
$$

Theorem 5.3. $X \subseteq \mathbb{P}^{r}$ is $(d-2+\ell)$-normal for all $\ell \geq m$.

Proof. There is an integer $k \geq 0$ such that $m=c_{k}+k$. Consider a filtration

$$
V_{k}:=V^{c_{k}} \subseteq V^{c_{k}-1} \subseteq \cdots \subseteq V^{0}=H^{0}\left(\mathcal{O}_{X}\left(D_{\text {out }}+k H\right)\right)
$$

by subspaces each having codimension one in the next. Note that each $V^{i}$ is a base point free subspace for $0 \leq i \leq c_{k}$. We have exact sequences

$$
\begin{gathered}
0 \longrightarrow M_{V^{i}} \longrightarrow V^{i} \otimes \mathcal{O}_{X} \longrightarrow \mathcal{O}_{X}\left(D_{\text {out }}+k H\right) \longrightarrow 0 \\
0 \longrightarrow M_{V^{i+1}} \longrightarrow M_{V^{i}} \longrightarrow \mathcal{O}_{X} \longrightarrow 0
\end{gathered}
$$

We first show that

$$
V^{c_{k}} \otimes H^{0}\left(X, \mathcal{O}_{X}\left(K_{X}+(n+c) H\right)\right) \longrightarrow H^{0}\left(X, \mathcal{O}_{X}(d-2+k+c)\right)
$$

is surjective for all $c \geq c_{k}$. For this purpose, it suffices to establish that

$$
H^{1}\left(X, M_{V^{c_{k}}} \otimes \mathcal{O}_{X}\left(K_{X}+(n+c) H\right)\right)=0 \text { for all } c \geq c_{k} .
$$

Note that $D_{\text {out }}+k H=K_{X}+(n+1) H-2 K_{X}+(d-2 n-3+k) H$ and $-2 K_{X}+(d-2 n-3+k) H$ are assumed to be nef. By Theorem 5.1, we have

$$
H^{j}\left(X, \wedge^{c_{k}+j} M_{V^{0}} \otimes \mathcal{O}_{X}\left(K_{X}+n H+c H\right)\right)=0 \text { for all } j \geq 1, c \geq c_{k} .
$$

Consider the following short exact sequence

$$
0 \longrightarrow \wedge^{p} M_{V^{i+1}} \longrightarrow \wedge^{p} M_{V^{i}} \longrightarrow \wedge^{p-1} M_{V^{i+1}} \longrightarrow 0 .
$$

Twisting by $\mathcal{O}_{X}\left(K_{X}+n H+c H\right)$ and taking cohomology sequence, it is easy to check that

$$
H^{j}\left(X, \wedge^{c_{1}+j} M_{V^{1}} \otimes \mathcal{O}_{X}\left(K_{X}+n H+c H\right)\right)=0 \text { for all } j \geq 1, c \geq c_{k}, c-1 \geq c_{1} \geq 0 .
$$

By an induction on $i$ and similar arguments, we can also show that

$$
H^{j}\left(X, \wedge^{c_{i}+j} M_{V^{i}} \otimes \mathcal{O}_{X}\left(K_{X}+n H+c H\right)\right)=0 \text { for all } j \geq 1, c \geq c_{k}, c-i \geq c_{i} \geq 0 .
$$

In particular, we get the cohomology vanishing (5.1). This implies that the multiplication map

$$
V_{k} \otimes H^{0}\left(X, \mathcal{O}_{X}\left(K_{X}+(n+c) H\right)\right) \rightarrow H^{0}\left(X, \mathcal{O}_{X}(d-2+k+c)\right)
$$

is surjective for all $c \geq c_{k}$. Now, the assertion follows from Lemma 5.2.

To prove Theorem D, we may assume that $X \subseteq \mathbb{P}^{r}$ is non-degenerate and $n, e \geq 2$. Then Theorem D follows from Theorem B and Theorem 5.3.

In view of Theorem 5.3, it is natural to ask when the divisor $-2 K_{X}+(d-2 n-3+k) H$ appeared in the definition of $m$ is nef. If $H$ is sufficiently positive (e.g., $H=\ell A$, where $A$ is a very ample divisor and $\ell \geq 2$ is an integer $)$, then $-2 K_{X}+(d-2 n-3) H$ is already nef. In general, we have the following.

Proposition 5.4. $-2 K_{X}+(d-2 n-3+k) H$ is nef for $k \geq d-1$. 
Proof. We write $-2 K_{X}+(d-2 n-3+k) H=2 D_{\text {out }}+(k-d+1) H$. Since $D_{\text {out }}$ is base point free by Proposition 2.5, the assertion follows.

To give a bound for $m$ in terms of $d, n, e$, it is necessary to control the codimension $c_{k}$. One approach is to use the hyperplane section method. For this purpose, we fix some notations. Let $Y \subseteq \mathbb{P}^{r-1}$ be a general hyperplane section of $X \subseteq \mathbb{P}^{r}$, which is a non-degenerate smooth projective variety of dimension $n-1$, codimension $e$, and degree $d$. Note that

$$
\left.D_{\text {out }}\right|_{Y}=-\left.\left(K_{X}+H\right)\right|_{Y}+\left.(d-(n-1)-2) H\right|_{Y}=-K_{Y}+\left.(d-(n-1)-2) H\right|_{Y} .
$$

By abuse of notation, we write $D_{\text {out }}$ for the double point divisor from outer projection of $Y \subseteq \mathbb{P}^{r-1}$ and $H$ for general hyperplane section of $Y \subseteq \mathbb{P}^{r-1}$. Let $V_{X}$ and $V_{Y}$ be subspaces of $H^{0}\left(X, \mathcal{O}_{X}\left(D_{\text {out }}\right)\right)$ and $H^{0}\left(Y, \mathcal{O}_{Y}\left(D_{\text {out }}\right)\right)$ spanned by geometric sections, respectively, and $c_{k}^{X}$ and $c_{k}^{Y}$ be the codimensions of the images of the maps $V_{X} \otimes H^{0}\left(\mathcal{O}_{X}(k)\right) \rightarrow H^{0}\left(X, \mathcal{O}_{X}\left(D_{\text {out }}+k H\right)\right)$ and $V_{Y} \otimes H^{0}\left(\mathcal{O}_{Y}(k)\right) \rightarrow H^{0}\left(Y, \mathcal{O}_{Y}\left(D_{\text {out }}+k H\right)\right)$, respectively, for any integer $k \geq 0$.

Note that there is a natural injective map $\left|V_{Y}\right| \hookrightarrow\left|V_{X}\right|$, which induces an injective map $V_{Y} \hookrightarrow V_{X}$. We then have the following:

(1) If $H^{1}\left(X, \mathcal{O}_{X}(k)\right)=H^{1}\left(X, \mathcal{O}_{X}(k+1)\right)=H^{1}\left(X, \mathcal{O}_{X}\left(D_{\text {out }}+k H\right)\right)=H^{2}\left(X, \mathcal{O}_{X}(k)\right)=$ $H^{1}\left(Y, \mathcal{O}_{Y}(k+1)\right)=0$ (these conditions are satisfied if $k \geq d-1$ ), then we have

$$
c_{k+1}^{X} \leq c_{k}^{X}+c_{k+1}^{Y} .
$$

(2) If $H^{1}\left(X, \mathcal{O}_{X}(\ell)\right)=H^{1}\left(X, \mathcal{O}_{X}\left(D_{\text {out }}+\ell H\right)\right)=H^{2}\left(X, \mathcal{O}_{X}(\ell)\right)=H^{1}\left(Y, \mathcal{O}_{Y}(\ell+1)\right)=0$ for $\ell \geq k$ and $M_{V_{X}} \otimes \mathcal{O}_{Y}$ is $(k+1)$-regular (these conditions are satisfied if $k \geq d-1$ and $\left.c_{k}^{Y}=0\right)$, then we have

$$
c_{k+1}^{X}<c_{k}^{X} \text { provided that } c_{k}^{X} \neq 0 .
$$

The proof is straightforward by the standard vector bundle techniques, so we leave the details to the interested readers.

Suppose now that $c_{\ell}^{Y}=0$ for some integer $\ell \geq d-1$. From now on, we write $c_{k}=c_{k}^{X}$. Then, for $k \geq \ell$, we have

$$
c_{k+1}+k+1 \leq c_{k}+k
$$

provided that $c_{k} \neq 0$. Since $-2 K_{X}+(d-2 n-3+k) H$ is nef for $k \geq d-1$ by Proposition 5.4 , calculating $m$ is almost equivalent to finding the minimum $k$ such that $c_{k}=0$ for $k \geq d-1$. In this respect, we show the following.

Proposition 5.5. We have $c_{k}=0$ for $k \geq n(d-3)$.

Proof. We can take a subspace $W$ of $V$ generated by $n+1$ general geometric sections so that $W$ is also base point free. We have the following exact sequence

$$
0 \longrightarrow M_{W} \longrightarrow W \otimes \mathcal{O}_{X} \longrightarrow \mathcal{O}_{X}\left(D_{\text {out }}\right) \longrightarrow 0 \text {. }
$$

Since $M_{W}$ is a rank $n$ vector bundle, $M_{W} \simeq \wedge^{n-1} M_{W}^{*} \otimes \mathcal{O}_{X}\left(-D_{\text {out }}\right)$. We shall show that

$$
H^{1}\left(X, \wedge^{n-1} M_{W}^{*} \otimes \mathcal{O}_{X}\left(-D_{\text {out }}+k H\right)\right)=0 \text { for } k \geq n(d-3) .
$$

Now, since $(X, \mathcal{O}(1)) \neq\left(\mathbb{P}^{n}, \mathcal{O}_{\mathbb{P}^{n}}(1)\right)$, it follows from the main result of $[\mathrm{E}]$ that $K_{X}+n H$ is base point free. Notice that $k \geq n(d-3) \geq j d-2 j-n$ for $1 \leq j \leq n-1$. We write

$$
-j D_{\text {out }}+k H=K_{X}+(j-1)\left(K_{X}+n H\right)+(k-j d+2 j+n) H .
$$

Then, by Kodaira vanishing theorem, we have

$$
H^{i}\left(X, \mathcal{O}_{X}\left(-j D_{\text {out }}+k H\right)\right)=0 \text { for } i>0,
$$


For each $1 \leq i \leq n$, from the following exact sequence

$$
0 \rightarrow \wedge^{n-i} M_{W}^{*} \otimes \mathcal{O}_{X}\left(-D_{\text {out }}\right) \rightarrow \wedge^{n-i+1} W^{*} \otimes \mathcal{O}_{X} \rightarrow \wedge^{n-i+1} M_{W}^{*} \rightarrow 0,
$$

we obtain

$H^{i-1}\left(\wedge^{n-(i-1)} M_{W}^{*} \otimes \mathcal{O}_{X}\left(-(i-1) D_{\text {out }}+k H\right)\right)=H^{i}\left(\wedge^{n-i} M_{W}^{*} \otimes \mathcal{O}_{X}\left(-i D_{\text {out }}+k H\right)\right)$ for $2 \leq i \leq n$.

This implies that

$$
H^{1}\left(X, \wedge^{n-1} M_{W}^{*} \otimes \mathcal{O}_{X}\left(-D_{\text {out }}+k H\right)\right)=H^{n}\left(X, \mathcal{O}_{X}\left(-n D_{\text {out }}+k H\right)\right)=0 .
$$

This proves the assertion.

Remark 5.6. There are only a few cases such that $n(d-3)<d-1$, and the regularity conjecture holds for all such cases. Thus we may assume that $n(d-3) \geq d-1$. Then Propositions 5.4 and 5.5 imply that $m \leq n(d-3)$. By Theorem $\mathrm{D}$, we obtain

$$
\operatorname{reg}(X) \leq(n+1)(d-3)+2 .
$$

which is unfortunately weaker than Theorem C. In the proof of Proposition 5.5, only $n+1$ general geometric sections in $V$ are used. We expect that a systematic approach to use of all geometric sections in $V$ would lead us to a better bound for $m$ as well as $\operatorname{reg}(X)$.

5.2. Inner projection case. The cohomological method in Subsection 5.1 can be directly generalized to the inner projection case under suitable assumptions. Throughout this subsection, we use the following notations. Let $X \subseteq \mathbb{P}^{r}$ be a non-degenerate smooth projective variety of dimension $n$, codimension $e$, and degree $d$, and $H$ be its hyperplane section. As in Subsection 5.1, we may assume that $n, e \geq 2$. We also need the following conditions:

(1) Assume that $X \subseteq \mathbb{P}^{r}$ is neither a scroll over a curve, the second Veronese surface in $\mathbb{P}^{5}$, nor a Roth variety. Then we can consider the double point divisor from inner projection

$$
D_{i n n}=-K_{X}+(d-n-e-1) H,
$$

which is semiample by Theorem 2.7. Let $V^{\prime}:=V_{i n n}$ be the linear subspace of $H^{0}\left(\mathcal{O}_{X}\left(D_{i n n}\right)\right)$ spanned by geometric sections.

(2) Assume that $V^{\prime}$ is base point free. Recall that $\operatorname{Bs}\left(\left|V^{\prime}\right|\right) \subseteq \mathcal{C}(X)$. Thus if $\mathcal{C}(X)=\emptyset$, then $V^{\prime}$ is base point free (see [No1, Corollary 3] for examples with $\mathcal{C}(X)=\emptyset$ ).

(3) Assume that the multiplication map

$$
W_{i} \otimes H^{0}\left(X, \mathcal{O}_{X}\left(K_{X}+(n-1) H\right)\right) \longrightarrow H^{0}\left(X, \mathcal{O}_{X}\left(K_{X}+(n-1+i) H\right)\right)
$$

is surjective for $i=1,2$, where $W_{k}$ is the image of the map $H^{0}\left(\mathbb{P}^{r}, \mathcal{O}_{\mathbb{P}^{r}}(k)\right) \rightarrow H^{0}\left(X, \mathcal{O}_{X}(k)\right)$ for any integer $k$. Since $\mathcal{O}_{X}\left(K_{X}+(n+1) H\right)$ is 0-regular with respect to $\mathcal{O}_{X}(1)$, it follows that the multiplication map

$$
W_{k} \otimes H^{0}\left(X, \mathcal{O}_{X}\left(K_{X}+(n-1) H\right)\right) \longrightarrow H^{0}\left(X, \mathcal{O}_{X}\left(K_{X}+(n-1+k) H\right)\right)
$$

is surjective for any integer $k \geq 0$.

We first prove the following, which is a counterpart of Lemma 4.1.

Lemma 5.7. Let $D \in\left|V^{\prime}\right|$ be an effective divisor on $X$. Then the natural restriction map

$$
H^{0}\left(\mathbb{P}^{r}, \mathcal{I}_{D \mid \mathbb{P}^{r}}(d-e-2+\ell)\right) \longrightarrow H^{0}\left(X, \mathcal{I}_{D \mid X}(d-e-2+\ell)\right)
$$

is surjective for any integer $\ell \leq 0$. 
Proof. As in Lemma 4.1, it is sufficient to show the assertion for a geometric divisor $D=D_{\text {inn }}(\Lambda)$ associated to a general inner projection $\pi=\pi_{\Lambda}: X \rightarrow \bar{X} \subseteq \mathbb{P}^{n+1}$ centered at a general linear subspace $\Lambda \subseteq \mathbb{P}^{r}$ meeting $X$ at general $(e-1)$ points on $X$. Let $x_{1}, \ldots, x_{e-1}$ be general points on $X$ such that $X \cap \Lambda=\left\{x_{1}, \ldots, x_{e-1}\right\}$, and $\sigma: \widetilde{X} \rightarrow X$ be the blow-up at $x_{1}, \ldots, x_{e-1}$ with exceptional divisors $E_{1}, \ldots, E_{e-1}$. Put $E:=E_{1}+\cdots+E_{e-1}$. Note that the birational morphism $\tilde{\pi}:=\sigma \circ \pi: \widetilde{X} \rightarrow \bar{X}$ is a resolution of singularities and has no exceptional divisor by our assumption. Let $\bar{H}$ be a general hyperplane section of $\bar{X} \subseteq \mathbb{P}^{n+1}$. Then we have $\widetilde{\pi}^{*} \bar{H}=\sigma^{*} H-E$. Let $\widetilde{D}$ be the non-isomorphic locus of $\widetilde{\pi}$ so that $\sigma(\widetilde{D})=D_{i n n}(\Lambda)$. By the birational double point formula ([L2, Lemma 10.2.8]), we have

$$
\widetilde{D} \sim-K_{\widetilde{X}}+(d-n-e-1) \widetilde{\pi}^{*} \bar{H} \sim \sigma^{*}\left(D_{i n n}\right)+(-d+e+2) E .
$$

The image $\bar{D}:=\overline{\widetilde{\pi}(\widetilde{D})}$ is a Weil divisor on $\bar{X}$. We then obtain the following diagram:

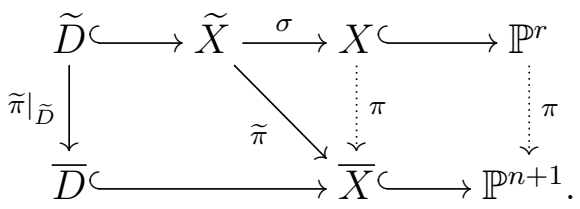

Note that $\mathcal{I}_{\bar{D} \mid \mathbb{P}^{n+1}}=\operatorname{adj}\left(\mathbb{P}^{n+1}, \bar{D}\right)$ is the adjoint ideal and $\mathcal{I}_{\bar{D} \mid \bar{X}} \simeq \widetilde{\pi}_{*} \mathcal{I}_{\widetilde{D} \mid \widetilde{X}}=\widetilde{\pi}_{*} \mathcal{O}_{\widetilde{X}^{\prime}}(-\widetilde{D})$ (see [L2, Proposition 9.3.48]). Consider the following short exact sequence

$$
0 \longrightarrow \mathcal{I}_{\bar{X} \mid \mathbb{P}^{n+1}} \longrightarrow \mathcal{I}_{\bar{D} \mid \mathbb{P}^{n+1}} \longrightarrow \mathcal{I}_{\bar{D} \mid \bar{X}} \longrightarrow 0 .
$$

For any integer $\ell$, we have

$$
(d-e-2+\ell) \widetilde{\pi}^{*} \bar{H}-\widetilde{D} \sim K_{\widetilde{X}}+(n-1+\ell) \widetilde{\pi}^{*} \bar{H} \sim \sigma^{*}\left(K_{X}+(n-1+\ell) H\right)-\ell E .
$$

Thus, for any integer $\ell \leq 0$, we obtain

$$
H^{0}\left(\bar{X}, \mathcal{I}_{\bar{D} \mid \bar{X}}(d-e-2+\ell)\right)=H^{0}\left(X, \mathcal{O}_{X}\left(K_{X}+(n-1+\ell) H\right)\right)=H^{0}\left(X, \mathcal{I}_{D \mid X}(d-e-2+\ell)\right) .
$$

We have the following commutative diagram:

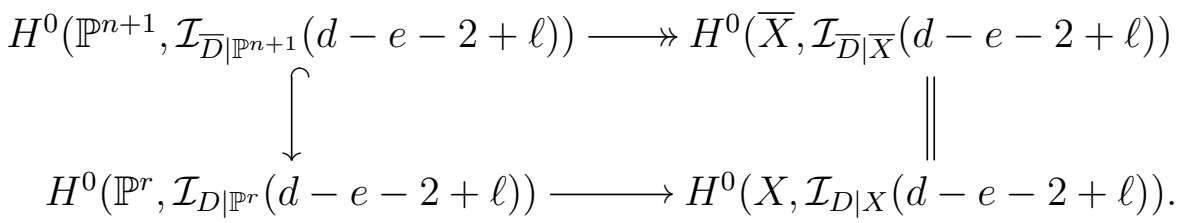

Since $\mathcal{I}_{\bar{X} \mid \mathbb{P}^{n+1}} \simeq \mathcal{O}_{\mathbb{P}^{n+1}}(-(d-e+1))$ and $H^{1}\left(\mathbb{P}^{n+1}, \mathcal{O}_{\mathbb{P}^{n+1}}(\ell)\right)=0$, it follows that the upper horizontal map is surjective. Thus the lower horizontal map is also surjective.

Remark 5.8. Unlike Lemma 4.1, we need to assume that $\ell \leq 0$ in Lemma 5.7. If $\ell \geq 1$, then we only have

$$
H^{0}\left(\bar{X}, \mathcal{I}_{\bar{D} \mid \bar{X}}(d-e-2+\ell)\right) \subseteq H^{0}\left(X, \mathcal{I}_{D \mid X}(d-e-2+\ell)\right),
$$

so we cannot deduce the surjectivity of the map

$$
H^{0}\left(\mathbb{P}^{r}, \mathcal{I}_{D \mid \mathbb{P}^{r}}(d-e-2+\ell)\right) \longrightarrow H^{0}\left(X, \mathcal{I}_{D \mid X}(d-e-2+\ell)\right) .
$$

For instance, consider a projected second Veronese embedding $X \subseteq \mathbb{P}^{4}$. We have $d=4$ and $e=2$. Let $A=\frac{1}{2} H$ be the ample generator of $\operatorname{Pic}(X)$. Then $D_{i n n} \sim A$. For any geometric divisor $D \in\left|D_{\text {inn }}\right|$, we see that $H^{0}\left(\mathbb{P}^{4}, \mathcal{I}_{D \mid \mathbb{P}^{4}}(1)\right) \rightarrow H^{0}\left(X, \mathcal{I}_{D \mid X}(1)\right)$ is not surjective. 
Let $V_{k}^{\prime}$ be the image of of the multiplication map

$$
V^{\prime} \otimes H^{0}\left(X, \mathcal{O}_{X}(k)\right) \longrightarrow H^{0}\left(X, \mathcal{O}_{X}\left(D_{i n n}+k H\right)\right)
$$

for any integer $k \geq 0$, and $c_{k}^{\prime}$ be the codimension of $V_{k}^{\prime}$ in $H^{0}\left(X, \mathcal{O}_{X}\left(D_{i n n}+k H\right)\right)$.

Lemma 5.9 (A generalized Mumford's lemma for inner projection). If the multiplication map

$$
V_{k}^{\prime} \otimes H^{0}\left(X, \mathcal{O}_{X}\left(K_{X}+(n-1) H\right)\right) \longrightarrow H^{0}\left(X, \mathcal{O}_{X}(d-e-2+k)\right)
$$

is surjective for some integer $k \geq 0$, then $X \subseteq \mathbb{P}^{r}$ is $(d-e-2+k)$-normal.

Proof. By considering the following commutative diagram

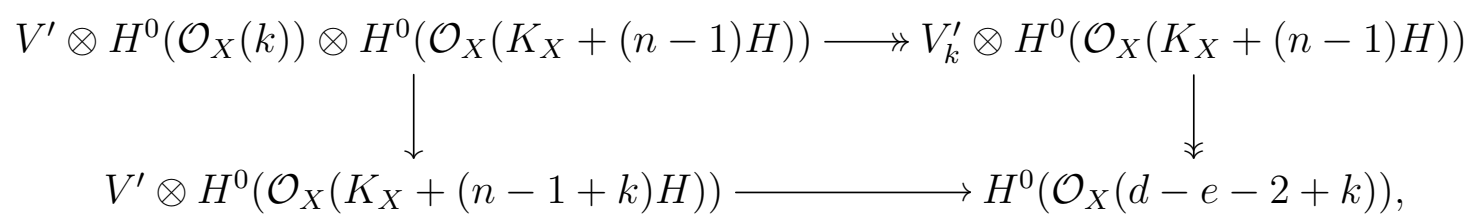

we see that the bottom map is surjective. Recall that the multiplication map

$$
W_{k} \otimes H^{0}\left(X, \mathcal{O}_{X}\left(K_{X}+(n-1) H\right)\right) \longrightarrow H^{0}\left(X, \mathcal{O}_{X}\left(K_{X}+(n-1+k) H\right)\right)
$$

is surjective. By Lemma 5.7, we have the map

$$
V^{\prime} \otimes H^{0}\left(X, \mathcal{O}_{X}\left(K_{X}+(n-1) H\right)\right) \longrightarrow W_{d-e-2} \subseteq H^{0}\left(X, \mathcal{O}_{X}(d-e-2)\right) .
$$

By considering the following commutative diagram

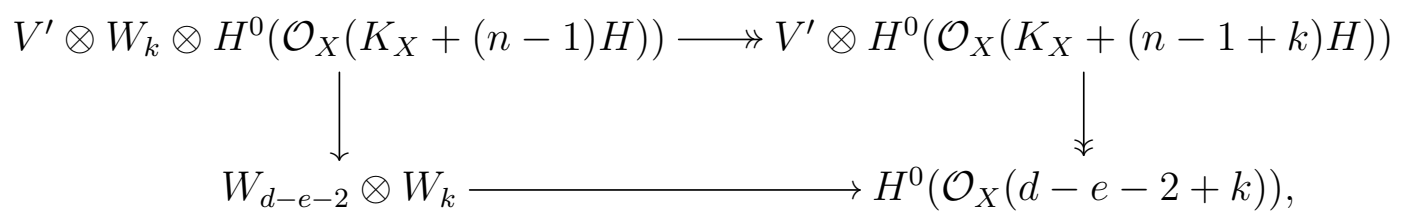

we see that the bottom map is surjective, and hence, the assertion follows.

As in the outer projection case, we set

$$
m^{\prime}:=\min \left\{c_{k}^{\prime}+k \mid-2 K_{X}+(d-2 n-e-2+k) H \text { is nef }\right\} .
$$

By the same argument in the proof of Theorem 5.3 using Lemma 5.9, we can prove the following theorem. We leave the details to the interested readers.

Theorem 5.10. $X \subseteq \mathbb{P}^{r}$ is $(d-e-2+\ell)$-normal for all $\ell \geq m^{\prime}$.

Now, Theorem E follows from Theorem B and Theorem 5.10.

\section{REFERENCES}

$[\mathrm{BM}]$ D. Bayer and D. Mumford, What can be computed in algebraic geometry?, Computational algebraic geometry and commutative algebra (Cortona, 1991), Sympos. Math., XXXIV, Cambridge Univ. Press, Cambridge (1993), 1-48.

[BEL] A. Bertram, L. Ein and R. Lazarsfeld, Vanishing theorems, a theorem of Severi, and the equations defining projective varieties, J. Amer. Math. Soc. 4 (1991), 587-602.

[B] D. C. Butler, Normal generation of vector bundles over a curve, J. Differential Geom. 39 (1994), 1-34.

[C] G. Castelnuovo, Sui multipli di una serie lineare di gruppi di punti appartenente ad una curva algebrica Rend. Circ. Mat. Palermo 7 (1893), 89-110.

[dFE] T. de Fernex and L. Ein, A vanishing theorem for log canonical pairs, Amer. J. Math. 132 (2010), 1205-1221. 
[E] L. Ein, The ramification divisors for branched coverings of $\mathbb{P}_{k}^{n}$, Math. Ann. 261 (1982), $483-485$.

[EL] L. Ein and R. Lazarsfeld, Syzygies and Koszul cohomology of smooth projective varieties of arbitrary dimension, Invent. Math. 111 (1993), 51-67.

[EG] D. Eisenbud and S. Goto, Linear free resolutions and minimal multiplicity, J. Algebra 88 (1984), 89-133.

$[\mathrm{EH}]$ D. Eisenbud and J. Harris, On varieties of minimal degree (a centennial account), Algebraic geometry, Bowdoin (Brunswick, Maine, 1985), Proc. Sympos. Pure Math. 46 (1987), Part 1, Amer. Math. Soc., Providence, RI, 3-13.

[F1] T. Fujita, On the structure of polarized manifolds with total deficiency one I, J. Math. Soc. Japan 32 (1980), 709-725.

[F2] T. Fujita, On the structure of polarized manifolds with total deficiency one II, J. Math. Soc. Japan 33 (1981), 415-434.

[GLP] L. Gruson, R. Lazarsfeld and C. Peskine, On a theorem of Castelnuovo, and the equations defining space curves, Invent. Math. 72 (1983), 491-506.

[Il] B. Ilic, Geometric properties of the double-point divisor, Trans. Amer. Math. Soc. 350 (1998), $1643-1661$.

[Io] P. Ionescu, Embedded projective varieties of small invariants, in Algebraic Geometry (Bucharest 1982), Lecture Notes in Math., 1056, Springer-Verlag, Berlin (1984), 142-186.

[IT] P. Ionescu and M. Toma, On very ample vector bundles on curves, Internat. J. Math. 8 (1997), 633-643.

[K1] S. Kwak, Castelnuovo regularity for smooth subvarieties of dimensions 3 and 4. J. Algebraic Geom. 7 (1998), 195-206.

[K2] S. Kwak, Castelnuovo-Mumford regularity bound for smooth threefolds in $\mathbb{P}^{5}$ and extremal examples, J. Reine Angew. Math. 509 (1999), 21-34.

[K3] S. Kwak, Generic projections, the equations defining projective varieties and Castelnuovo regularity, Math. Z. 234 (2000), 413-434.

[KP] S. Kwak and J. Park, Geometric properties of projective manifolds of small degree, Math. Proc. Cambridge Philos. Soc. 160 (2016), 257-277.

[L1] R. Lazarsfeld, A sharp Castelnuovo bound for smooth surfaces, Duke Math. J. 55 (1987), 423-429.

[L2] R. Lazarsfeld, Positivity in algebraic geometry I and II, A Series of Modern Surveys in Math. 48 and 49 (2004), Springer-Verlag, Berlin.

[MP] J. McCullough and I. Peeva, Counterexamples to the Eisenbud-Goto regularity conjecture, J. Amer. Math. Soc. 31 (2018), 473-496.

[M] D. Mumford, Varieties defined by quadratic equations, Questions on Algebraic Varieties (C.I.M.E., III Ciclo, Varenna, 1969) Edizioni Cremonese, Rome, 1970, 29-100.

[Ni] W. Niu, Castelnuovo-Mumford regularity bounds for singular surfaces, Math. Z. 280 (2015), $609-620$.

[NP1] W. Niu and J. Park, A Castelnuovo-Mumford regularity bound for scrolls, J. Algebra 488 (2017), 388-402.

[NP2] W. Niu and J. Park, A Castelnuovo-Mumford regularity bound for threefolds with rational singularities, preprint (2017).

[No1] A. Noma, Hypersurfaces cutting out a projective variety, Trans. Amer. Math. Soc. 362 (2010), $4481-4495$.

[No2] A. Noma, Generic inner projections of projective varieties and an application to the positivity of double point divisors, Trans. Amer. Math. Soc. 366 (2014), 4603-4623.

[No3] A. Noma, Projective varieties with nonbirational linear projections and applications, Trans. Amer. Math. Soc. 370 (2018), 2299-2320.

[P] H. Pinkham, A Castelnuovo bound for smooth surfaces, Invent. Math. 83 (1986), 321-332.

Department of Mathematical Sciences, KAiST, Daejeon, Korea

E-mail address: sjkwak@kaist.ac.kr

School of Mathematics, Korea Institute for Advanced Study, Seoul, Korea

E-mail address: parkjh13@kias.re.kr 